\title{
THE KERNELS AND CONTINUITY IDEALS OF HOMOMORPHISMS FROM $\mathcal{C}_{0}(\Omega)$
}

\author{
HUNG LE PHAM \\ (Received 29 October 2008; accepted 31 July 2009) \\ Communicated by G. A. Willis \\ In memoriam: Graham Robert Allan (1936-2007)
}

\begin{abstract}
We give a description of the continuity ideals and the kernels of homomorphisms from the algebras of continuous functions on locally compact spaces into Banach algebras. We also construct families of prime ideals satisfying a certain intriguing property in the algebras of continuous functions.
\end{abstract}

2000 Mathematics subject classification: primary 46H40; secondary 46J10.

Keywords and phrases: Banach algebra, algebra of continuous functions, automatic continuity, prime ideal.

\section{Introduction}

Let $\theta: A \rightarrow B$ be a homomorphism from a commutative Banach algebra $A$ into a Banach algebra $B$. The continuity ideal of $\theta$ is defined to be the ideal

$$
\mathcal{I}(\theta)=\{a \in A \mid \text { the map } b \mapsto \theta(a b), A \rightarrow B, \text { is continuous }\} ;
$$

this ideal contains every ideal $I$ in $A$ on which $\theta$ is continuous. If $A=\mathcal{C}_{0}(\Omega)$ for some locally compact space $\Omega$, then $\theta$ is continuous on $\mathcal{I}(\theta)$.

The aim of this paper is to characterize the ideals that are the kernels or the continuity ideals of homomorphisms from $\mathcal{C}_{0}(\Omega)$ into Banach algebras. This is, in some sense, a final piece of the picture of homomorphisms from $\mathcal{C}_{0}(\Omega)$ into Banach algebras.

The study of homomorphisms from $\mathcal{C}_{0}(\Omega)$ started with the theorem of Kaplansky [13] that every algebra norm on $\mathcal{C}_{0}(\Omega)$ majorizes the uniform norm. This essentially provides a description of all the continuous homomorphisms from $\mathcal{C}_{0}(\Omega)$ into Banach algebras.

Then Bade and Curtis [1] gave a detailed structural decomposition of discontinuous homomorphisms from $\mathcal{C}_{0}(\Omega)$ into Banach algebras. The following statement of their

This research was supported by a Killam Postdoctoral Fellowship and an honorary Pacific Institute for the Mathematical Sciences Postdoctoral Fellowship.

(C) 2010 Australian Mathematical Publishing Association Inc. 1446-7887/2010 \$16.00 
theorem also includes some improvements from [6, 17] (see also [3, 18], noting that other improvements of this theorem can be found in [12]); see Section 2 for notation.

THEOREM 1.1. Suppose that $\Omega$ is a locally compact space, and that $\theta$ is a discontinuous homomorphism from $\mathcal{C}_{0}(\Omega)$ into a Banach algebra $B$. Set $B_{0}=$ $\overline{\theta\left(\mathcal{C}_{0}(\Omega)\right)}$.

(i) The continuity ideal $\mathcal{I}(\theta)$ is the largest ideal of $\mathcal{C}_{0}(\Omega)$ on which $\theta$ is continuous.

(ii) There exists a nonempty finite subset $\left\{p_{1}, \ldots, p_{n}\right\}$ of $\Omega^{b}$ such that

$$
\bigcap_{i=1}^{n} J_{p_{i}} \subseteq \mathcal{I}(\theta) \subseteq \bigcap_{i=1}^{n} M_{p_{i}}
$$

(iii) There exists a continuous homomorphism $\mu: \mathcal{C}_{0}(\Omega) \rightarrow B_{0}$ such that

$$
B_{0}=\mu\left(\mathcal{C}_{0}(\Omega)\right) \oplus \operatorname{rad} B_{0}, \quad \mu\left(\bigcap_{i=1}^{n} M_{p_{i}}\right) \cdot \operatorname{rad} B_{0}=\{0\},
$$

and $\mu=\theta$ on a dense subalgebra of $\mathcal{C}_{0}(\Omega)$ containing $\mathcal{I}(\theta)$.

(iv) Set $v=\theta-\mu$. Then $v$ maps into $\operatorname{rad} B_{0}$, and the restriction of $v$ to $\bigcap_{i=1}^{n} M_{p_{i}}$ is a homomorphism $v^{\prime}$ onto a dense subalgebra of $\mathrm{rad} B_{0}$.

(v) There exist linear maps $v_{1}, \ldots, v_{n}: \mathcal{C}_{0}(\Omega) \rightarrow \operatorname{rad} B_{0}$ such that:

(a) $v=v_{1}+\cdots+v_{n}$;

(b) $v_{i}^{\prime}=\left.v_{i}\right|_{M_{p_{i}}}$ is a nonzero homomorphism, whenever $1 \leq i \leq n$;

(c) $v_{i}\left(\mathcal{C}_{0}(\Omega)\right) \cdot v_{j}\left(\mathcal{C}_{0}(\Omega)\right)=\{0\}$ whenever $1 \leq i \neq j \leq n$.

(vi) The ideals $\operatorname{ker} \theta$ and $\mathcal{I}(\theta)$ are always intersections of prime ideals; we have

$$
\operatorname{ker} \theta=\mathcal{I}(\theta) \cap \operatorname{ker} \mu \quad \text { and } \quad \mathcal{I}(\theta)=\operatorname{ker} \nu^{\prime}=\bigcap_{i=1}^{n} \operatorname{ker} \nu_{i}^{\prime} .
$$

For brevity, we define a radical homomorphism to be a homomorphism into a radical Banach algebra. The above result points out the important roles of prime ideals and of radical homomorphisms as building blocks for general (discontinuous) homomorphisms from $\mathcal{C}_{0}(\Omega)$.

In the 1970s, Dales [2] and Esterle [6-8] independently proved that, assuming the continuum hypothesis, every ideal $I$ which is the intersection of a finite number of nonmodular prime ideals in $\mathcal{C}_{0}(\Omega)$ and such that $\left|\mathcal{C}_{0}(\Omega) / I\right|=\mathfrak{c}$ is the kernel of a radical homomorphism from $\mathcal{C}_{0}(\Omega)$ (for more details see [3]).

In fact, for some spaces $\Omega$, the kernels of radical homomorphisms from $\mathcal{C}_{0}(\Omega)$ are always finite intersections of nonmodular prime ideals [6, 15]. However, in [15], we showed that for most metrizable, noncompact, locally compact spaces $\Omega$, for example $\mathbb{R}$, there exist radical homomorphisms from $\mathcal{C}_{0}(\Omega)$ whose kernels are not the intersection of any finite number of prime ideals.

In this paper, we show that the kernels of radical homomorphisms from $\mathcal{C}_{0}(\Omega)$ are always intersections of (relatively) compact families of nonmodular prime 
ideals (Theorem 6.7(i)). In fact, we prove a more general result for continuity ideals of homomorphisms from Banach algebras into commutative Banach algebras (Corollary 4.11).

For the converse direction, we shall prove that, assuming the continuum hypothesis, every ideal $I$ that is the intersection of a relatively compact family $\mathfrak{P}$ of nonmodular prime ideals and is such that $\left|\mathcal{C}_{0}(\Omega) / I\right|=\mathfrak{c}$ and every chain in the closure of $\mathfrak{P}$ is countable (thus, in particular, when $\mathfrak{P}$ itself is countable), is the kernel of a radical homomorphism from $\mathcal{C}_{0}(\Omega)$. A similar result holds for continuity ideals of homomorphisms from $\mathcal{C}_{0}(\Omega)$ into Banach algebras: see Section 6. We conjecture that the countability condition in these converses is redundant (see the last paragraphs of Section 7).

REMARK 1.2. It was proved by Solovay and Woodin that the existence of discontinuous homomorphisms from $\mathcal{C}_{0}(\Omega)$ is not a theorem of Zermelo-Fraenkel set theory with the axiom of choice (see [4] for more details). All results that require the continuum hypothesis will be marked with $(\mathrm{CH})$.

In the final section, we shall construct nontrivial examples of (relatively) compact families of prime ideals in $\mathcal{C}_{0}(\Omega)$ for metrizable locally compact spaces $\Omega$ with infinite limit level (Theorem 7.5). This, in particular, shows the complexity of the prime ideal structure of $\mathcal{C}_{0}(\Omega)$ even for countable compact subspaces $\Omega$ of $\mathbb{R}$.

\section{Preliminary definitions and notation}

Let $A$ be a commutative algebra. The (conditional) unitization $A^{\#}$ of $A$ is defined as the algebra $A$ itself if $A$ is unital, and as $A$ with identity adjoined otherwise. The identity of $A^{\#}$ is denoted by $\mathbf{e}_{A}$.

A prime ideal or semiprime ideal in $A$ must be a proper ideal. However, we consider $A$ itself as the intersection of the empty collection of prime ideals.

Let $I$ be an ideal in $A$. Define the prime radical $\sqrt{I}$ of $I$ to be the intersection of all the prime ideals in $A$ containing $I$, so that

$$
\sqrt{I}=\left\{a \in A \mid a^{n} \in I \text { for some } n \in \mathbb{N}\right\} .
$$

For each element $a \in A^{\#}$, define the quotient of I by $a$ to be the ideal

$$
I: a=\{b \in A \mid a b \in I\} .
$$

Clearly $I \subseteq I: a$.

For the definition of universal algebras, see [3, Definition 5.7.8]. For example, the integral domain $L^{1}\left(\mathbb{R}^{+}, \omega\right)$ is universal for each radical weight $\omega$ bounded near the origin [3, Theorem 5.7.25]. Indeed, the class of universal, commutative, radical Banach algebras has been characterized in [10] (see also [3, Theorem 5.7.28]).

For a discussion of the theory of the algebras of continuous functions, see any of $[3,5,11]$. Here we just give some facts that are needed in our discussion. 
Let $\Omega$ be a locally compact space; the convention is that locally compact spaces and compact spaces are Hausdorff and nonempty. The one-point compactification of $\Omega$ is denoted by $\Omega^{b}$. Denote by $\mathcal{C}_{0}(\Omega)$ the algebra of (complex-valued) continuous functions that vanish at infinity on $\Omega$. Denote by $\mathcal{C}_{c}(\Omega)$ the subalgebra of $\mathcal{C}_{0}(\Omega)$ consisting of functions with compact support. For all $p \in \Omega$, define

$$
\begin{gathered}
J_{p}=\left\{f \in \mathcal{C}_{0}(\Omega) \mid f \text { is zero on a neighbourhood of } p\right\}, \\
M_{p}=\left\{f \in \mathcal{C}_{0}(\Omega) \mid f(p)=0\right\} .
\end{gathered}
$$

When $p$ is the point (at infinity) adjoined to $\Omega$ to obtain $\Omega^{b}$, we also set

$$
J_{p}=\mathcal{C}_{c}(\Omega) \quad \text { and } \quad M_{p}=\mathcal{C}_{0}(\Omega) .
$$

For each prime ideal $P$ in $\mathcal{C}_{0}(\Omega)$, there exists a unique point $p \in \Omega^{b}$ such that $J_{p} \subseteq P \subseteq M_{p}$, and we say that $P$ is supported at the point $p$. It can be seen that $P$ is modular if and only if its support point belongs to $\Omega$.

We shall frequently use the important fact that, for each prime ideal $P$ in $\mathcal{C}_{0}(\Omega)$, the set of prime ideals containing $P$ is a chain with respect to the inclusion relation.

For each function $f$ continuous on $\Omega$, the zero set of $f$ is denoted by $\mathbf{Z}(f)$. The set of zero sets of continuous functions on $\Omega$ is denoted by $\mathbf{Z}[\Omega]$.

A $z$-filter $\mathcal{F}$ on $\Omega$ is a nonempty proper subset of $\mathbf{Z}[\Omega]$ such that:

(i) $Z_{1} \cap Z_{2}$ belongs to $\mathcal{F}$ whenever both $Z_{1}$ and $Z_{2}$ belong to $\mathcal{F}$;

(ii) if $Z_{1} \in \mathcal{F}, Z_{2} \in \mathbf{Z}[\Omega]$ and $Z_{1} \subseteq Z_{2}$, then $Z_{2}$ also belongs to $\mathcal{F}$.

Each $z$-filter $\mathcal{F}$ corresponds to an ideal

$$
\{f \in \mathcal{C}(\Omega) \mid \mathbf{Z}(f) \in \mathcal{F}\}
$$

denoted by $\mathbf{Z}^{-1}[\mathcal{F}]$.

Let $\kappa$ be an infinite cardinal and let $\mathcal{U}$ be a free ultrafilter on $\kappa$. Define $M_{\mathcal{U}}$ to be the maximal ideal of $\mathbb{C}^{\kappa}$ consisting of all elements $f \in \mathbb{C}^{\kappa}$ such that $\{\sigma \in \kappa \mid f(\sigma)=0\} \in$ $\mathcal{U}$. The field $\mathbb{C}^{\kappa} / M_{\mathcal{U}}$ is called an ultrapower, and is denoted by $\mathbb{C}^{\kappa} / \mathcal{U}$. An element $f+M_{\mathcal{U}} \in \mathbb{C}^{\kappa} / \mathcal{U}$ is an infinitesimal if

$$
\{\sigma \in \kappa|| f(\sigma) \mid<\varepsilon\} \in \mathcal{U} \quad \forall \varepsilon>0 .
$$

The subalgebra of infinitesimals of $\mathbb{C}^{\kappa} / \mathcal{U}$ is denoted by $\left(\mathbb{C}^{\kappa} / \mathcal{U}\right)^{\circ}$.

For a well-ordered set $\Lambda$, denote by o $\Lambda$ ) the ordinal order isomorphic to $\Lambda$.

\section{Relatively compact families of prime ideals}

In this section, let $A$ be a commutative algebra.

Definition 3.1 [15, Definition 3.1]. An indexed family $\left(P_{i}\right)_{i \in S}$ of prime ideals in $A$ is pseudofinite if $a \in P_{i}$ for all but finitely many $i \in S$ whenever $a \in \bigcup_{i \in S} P_{i}$. 
For a pseudofinite family ( $P_{i}: i \in S$ ) of prime ideals where $S$ is infinite, it is obvious that $\bigcup_{i \in T} P_{i}=\bigcup_{i \in S} P_{i}$ is either a prime ideal in $A$ or the whole of $A$ for each infinite subset $T$ of $S$.

Definition 3.2. A family $\mathfrak{C}$ of prime ideals in $A$ is relatively compact if every sequence of prime ideals in $\mathfrak{C}$ contains a pseudofinite subsequence. The family $\mathfrak{C}$ is compact if it is relatively compact and contains the union of all of its pseudofinite sequences.

Obviously, the union of finitely many pseudofinite families is relatively compact. In the rest of this section, we shall indeed relate our notion of compactness to the usual meaning of this terminology.

Denote by $\Pi$ the set of prime ideals in $A$. For all $a_{1}, a_{2}, \ldots, a_{m}$, and $b$ in $A$, define

$$
\mathcal{U}_{a_{1}, \ldots, a_{m}}^{b}=\left\{P \in \Pi \mid \text { all } a_{i} \in P \text { and } b \notin P\right\} .
$$

Then the collection of all such sets $\mathcal{U}_{a_{1}, \ldots, a_{m}}^{b}$ is a base for a topology $\tau$. Indeed, by the primeness, we have

$$
\mathcal{U}_{a_{1}, \ldots, a_{m}, c_{1}, \ldots, c_{n}}^{b d}=\mathcal{U}_{a_{1}, \ldots, a_{m}}^{b} \cap \mathcal{U}_{c_{1}, \ldots, c_{n}}^{d}
$$

It is also easy to see that $\tau$ is Hausdorff. We claim that $\mathcal{U}_{0}^{u}$ is $\tau$-compact for all $u \in A$. Indeed, we see that $\left\{\mathcal{U}_{a}^{u}, \mathcal{U}_{0}^{a} \mid a \in A\right\}$ is a subbasis for the relative $\tau$-topology on $\mathcal{U}_{0}^{u}$, so by Alexander's lemma, we need only show that each cover of $\mathcal{U}_{0}^{u}$ by sets in this subbasis has a finite subcover. Let $E, F$ be subsets of $A$ such that

$$
\mathcal{U}_{0}^{u}=\bigcup_{a \in E} \mathcal{U}_{a}^{u} \cup \bigcup_{b \in F} \mathcal{U}_{0}^{b}
$$

Set $S=\left\{u^{m} a_{1} \cdots a_{n} \mid m, n \in \mathbb{N}, a_{1}, \ldots, a_{n} \in E\right\}$, and let $I$ be the ideal generated by $F$. Assume towards a contradiction that $S \cap I=\emptyset$. Then since $S$ is closed under multiplication, there exists a prime ideal $P$ such that $P \supseteq I$ and $P \cap S=\emptyset$; this implies that $P \in \mathcal{U}_{0}^{u}$ but $P \notin \bigcup_{a \in E} \mathcal{U}_{a}^{u} \cup \bigcup_{b \in F} \mathcal{U}_{0}^{b}$, a contradiction. Thus $S \cap I \neq \emptyset$, so there exist $k, m, n \in \mathbb{N}, a_{1}, \ldots, a_{m} \in E, b_{1}, \ldots, b_{n} \in F$, and $c_{1}, \ldots, c_{n} \in A$ such that

$$
u^{k} a_{1} \cdots a_{m}=b_{1} c_{1}+\cdots+b_{n} c_{n} .
$$

We can then deduce that

$$
\mathcal{U}_{0}^{u}=\bigcup_{i=1}^{m} \mathcal{U}_{a_{i}}^{u} \cup \bigcup_{j=1}^{n} \mathcal{U}_{0}^{b_{j}}
$$

Thus $\tau$ is locally compact.

The one-point compactification of $(\Pi, \tau)$ can be considered as the set $\Pi \cup\{A\}$; a basis of neighbourhood for $A$ is given by the collection of all

$$
\mathcal{U}_{a_{1}, \ldots, a_{m}}=\{A\} \cup\left\{P \in \Pi \mid \text { all } a_{i} \in P\right\} \quad \text { where } a_{1}, a_{2}, \ldots, a_{m} \in A .
$$


Proposition 3.3. Let $A$ be a commutative algebra. Denote by $\Pi$ the set of prime ideals in $A$. Define a topology $\tau$ as above. Then $(\Pi, \tau)$ is a totally disconnected locally compact space, and every [relatively] compact family of prime ideals in $A$ is a [relatively] sequentially $\tau$-compact subset of $\Pi \cup\{A\}$.

PROOF. It remains to prove the last assertion. We claim that a pseudofinite sequence $\left(P_{n}\right)$ of prime ideals in $A$ is $\tau$-convergent in $\Pi \cup\{A\}$. In fact, set $P=\bigcup_{n=1}^{\infty} P_{n}$. Then either $P \in \Pi$ or $P=A$, and in both cases $\left(P_{n}\right) \tau$-converges to $P$.

REMARK 3.4. If $A$ is either unital or $\mathcal{C}_{0}(\Omega)$ for some locally compact space $\Omega$, then the union $\bigcup_{n=1}^{\infty} P_{n}$ is in fact a prime ideal in $A$, for each pseudofinite sequence $\left(P_{n}\right)$ of prime ideals in $A$. Thus in the above proposition, we can replace $\Pi \cup\{A\}$ by $\Pi$.

REMARK 3.5. Let us instead consider a topology $\sigma$ on $\Pi \cup\{A\}$ generated by

$$
\mathcal{U}_{a_{1}, \ldots, a_{m}}^{Q}=\left\{P \in \Pi \cup\{A\} \mid P \subseteq Q \text { and all } a_{i} \in P\right\},
$$

where $Q$ is either a semiprime ideal in $A$ or $A$ itself, and $a_{1}, \ldots, a_{m} \in Q$. Then a sequence of prime ideals in $A$ is pseudofinite if and only if it is convergent in $(\Pi \cup\{A\}, \sigma)$, and so a family of prime ideals in $A$ is [relatively] compact if and only if it is [relatively] sequentially compact in $(\Pi \cup\{A\}, \sigma)$. However, in general, $\sigma$ is neither Hausdorff nor locally compact. In the case where $A=\mathcal{C}_{0}(\Omega)$, then $(\Pi \cup\{A\}, \sigma)$ is Hausdorff (but not locally compact).

\section{Homomorphisms from general commutative Banach algebras}

Let $\theta: A \rightarrow B$ be a homomorphism from a commutative Banach algebra $A$ into a Banach algebra $B$. Let $\left(a_{n}: n \in \mathbb{N}\right)$ be a sequence in $A$. Then

$$
\mathcal{I}(\theta): a_{1} a_{2} \cdots a_{n} \subseteq \mathcal{I}(\theta): a_{1} a_{2} \cdots a_{n+1} \quad \forall n \in \mathbb{N} .
$$

It follows easily from the stability lemma (see [3, Corollary 5.2.7] or [18, Lemma 1.6] for the statement and proof) that there exists $n_{0} \in \mathbb{N}$ such that

$$
\mathcal{I}(\theta): a_{1} a_{2} \cdots a_{n}=\mathcal{I}(\theta): a_{1} a_{2} \cdots a_{n+1} \quad \forall n \geq n_{0}
$$

Thus $\mathcal{I}(\theta)$ is an abstract continuity ideal in the following sense.

Definition 4.1. Let $A$ be a commutative algebra. An ideal $I$ is an abstract continuity ideal if, for each sequence $\left(a_{n}\right)$ in $A$, there exists $n_{0} \in \mathbb{N}$ such that

$$
I: a_{1} a_{2} \cdots a_{n}=I: a_{1} a_{2} \cdots a_{n+1} \quad \forall n \geq n_{0} .
$$

PROPOSITION 4.2. Let $\mathfrak{P}$ be a relatively compact family of prime ideals in a commutative algebra $A$. Then $\bigcap_{P \in \mathfrak{P}} P$ is an abstract continuity ideal in $A$. 
Proof. Set $I=\bigcap_{P \in \mathfrak{P}} P$. Assume towards a contradiction that $I$ is not an abstract continuity ideal. Then there exists a sequence $\left(a_{n}\right)$ in $A$ such that

$$
I: a_{1} a_{2} \cdots a_{n} \subset I: a_{1} a_{2} \cdots a_{n+1} \quad \forall n \in \mathbb{N} .
$$

For all $n$, we see that

$$
I: a_{1} \cdots a_{n}=\bigcap_{\substack{P \in \mathfrak{P} \\ a_{1} \cdots a_{n} \notin P}} P .
$$

Thus it follows that there exists $P_{n} \in \mathfrak{P}$ such that $a_{1} \cdots a_{n} \notin P_{n}$ but $a_{1} \cdots a_{n+1} \in P_{n}$. The relative compactness implies that there exist $n_{1}, n_{2}, \ldots$, such that $n_{1}<n_{2}<\ldots$ and $\left(P_{n_{i}}\right)$ is pseudofinite. However, we see that $a_{1} a_{2} \cdots a_{n_{2}} \in P_{n_{1}}$, but $a_{1} a_{2} \cdots a_{n_{2}} \notin$ $P_{n_{i}}$ for all $i \geq 2$; this contradicts the pseudofiniteness.

The remainder of this section is devoted to a converse of the above proposition. Let $I$ be an abstract continuity ideal of a commutative algebra $A$. Denote by $\mathfrak{P}$ the set of prime ideals of the form $I: a$ for some $a \in A$. The following is a generalization of [15, Lemma 4.3].

LEMMA 4.3. For each cardinal $\kappa \leq|\mathfrak{P}|$, there exists a subfamily $\mathcal{G} \subseteq \mathfrak{P}$ with the properties that $|\mathcal{G}| \geq \kappa$ and that $|\{P \in \mathcal{G} \mid a \notin P\}|<\kappa$ for all $a \in \bigcup_{P \in \mathcal{G}} P$.

PRoOf. For each $a \in A \cup\left\{\mathbf{e}_{A}\right\}$, let $\mathfrak{P}_{a}$ be the set of prime ideals of the form $I: a b$ for some $b \in A$. We claim that there exists $a_{0} \in A \cup\left\{\mathbf{e}_{A}\right\}$ such that $\left|\mathfrak{P}_{a_{0}}\right| \geq \kappa$ and such that either $\left|\mathfrak{P}_{a_{0} a}\right|<\kappa$ or $I: a_{0} a=I: a_{0}$, for each $a \in A$. Indeed, assume that the contrary holds. Then, since $\left|\mathfrak{P}_{\mathbf{e}_{A}}\right| \geq \kappa$, by induction, there exists a sequence $\left(a_{n}\right) \subseteq A$ such that $\left|\mathfrak{P}_{a_{1} \cdots a_{n}}\right| \geq \kappa$ and such that

$$
I: a_{1} \cdots a_{n} \subset I: a_{1} \cdots a_{n+1} \quad \forall n \in \mathbb{N}
$$

This contradicts the definition of an abstract continuity ideal. Hence the claim holds.

Put $\mathcal{G}=\mathfrak{P}_{a_{0}}$; this obviously satisfies $|\mathcal{G}| \geq \kappa$. Suppose that $a \in A$ and that $\mathcal{G}^{\prime}=$ $\{P \in \mathcal{G} \mid a \notin P\}$ has cardinality at least $\kappa$. Then, for each $P \in \mathcal{G}^{\prime}$, we have $P: a=P$ because $a \notin P$. Thus $\mathcal{G}^{\prime} \subseteq \mathfrak{P}_{a_{0} a}$, and hence $\left|\mathfrak{P}_{a_{0} a}\right| \geq \kappa$. Therefore, by the claim, we must have $I: a_{0} a=I: a_{0}$. We now show that $\mathcal{G}^{\prime}=\mathcal{G}$. Assume towards a contradiction that $\mathcal{G}^{\prime} \neq \mathcal{G}$, and take $P \in \mathcal{G} \backslash \mathcal{G}^{\prime}$, say $P=I: a_{0} a_{1}$ for some $a_{1} \in A$. Then $a_{1} \in I: a_{0} a=I: a_{0}$ since $a \in P$, so that $a_{0} a_{1} \in I$. This implies that $P=A$, a contradiction. This proves that $\mathcal{G}$ has the desired property.

LEMMA 4.4. The prime radical $\sqrt{I}$ of the abstract continuity ideal $I$ is the intersection of the prime ideals in $\mathfrak{P}$.

PROOF. This is based on the commutative prime kernel theorem due to Sinclair (see [3, Theorem 5.3.15] or [18, Theorem 11.4]; the proof in [15, Lemma 4.1] works almost verbatim). 
LEMMA 4.5. Every element in $\mathfrak{P}$ contains a minimal element.

PROOF. Assume towards a contradiction that there is a sequence $\left(P_{n}=I: f_{n}\right) \subseteq \mathfrak{P}$ such that

$$
P_{1} \supset P_{2} \supset \cdots \supset P_{n} \supset \cdots
$$

For all $n$, choose $a_{n} \in A$ such that $a_{n} \in P_{n} \backslash P_{n+1}$. Then we see that $a_{1} \cdots a_{n} f_{n} \in I$ but $a_{1} \cdots a_{n} f_{n+1} \notin I$. Thus

$$
I: a_{1} a_{2} \cdots a_{n} \subset I: a_{1} a_{2} \cdots a_{n+1} \quad \forall n \in \mathbb{N}
$$

this contradicts $I$ being an abstract continuity ideal.

Lemma 4.6. Let $P$ be in $\mathfrak{P}$. Then there exists $a \in A \backslash P$ such that $a \in Q$ for all $Q \in \mathfrak{P}$ with $Q \nsubseteq P$.

Proof. Assume that the contrary holds. Pick $a_{1} \notin P$. Suppose that we have already picked $a_{1}, \ldots, a_{n} \notin P$. By the assumption, we can find $Q_{n} \in \mathfrak{P}$ such that $a_{1} \ldots a_{n} \notin$ $Q_{n}$ and $Q_{n} \nsubseteq P$. We can then choose $a_{n+1} \in Q_{n} \backslash P$. The induction can be continued. We see that $\left(a_{n}\right),\left(Q_{n}\right)$ as constructed satisfy $a_{1} \cdots a_{n} \notin Q_{n}$ but $a_{1} \cdots a_{n+1} \in Q_{n}$ for all $n \in \mathbb{N}$. Let $Q_{n}=I: f_{n}$. Then we see that $f_{n} \in I: a_{1} a_{2} \cdots a_{n+1} \backslash I: a_{1} a_{2} \cdots a_{n}$; this contradicts $I$ being an abstract continuity ideal.

LeMma 4.7. Let $\left\{P_{\alpha} \mid \alpha \in S\right\}$ be a subfamily of $\mathfrak{P}$. Then $\bigcap_{\alpha \in S} P_{\alpha}$ is also an abstract continuity ideal.

PROOF. Assume that the contrary holds. Then there exists $\left(a_{n}\right)$ such that

$$
\left(\bigcap_{\alpha \in S} P_{\alpha}\right): a_{1} a_{2} \cdots a_{n} \subset\left(\bigcap_{\alpha \in S} P_{\alpha}\right): a_{1} a_{2} \cdots a_{n+1} \quad \forall n \in \mathbb{N} .
$$

Choose $b_{n} \in A$ such that $a_{1} \cdots a_{n} b_{n} \notin \bigcap_{\alpha \in S} P_{\alpha}$ but $a_{1} \cdots a_{n+1} b_{n} \in \bigcap_{\alpha \in S} P_{\alpha}$ for all $n$. Then choose $\alpha_{n} \in S$ such that $a_{1} \cdots a_{n} b_{n} \notin P_{\alpha_{n}}$. We have $P_{\alpha_{n}}=I: f_{\alpha_{n}}$ for some $f_{\alpha_{n}} \in A$. We see that $a_{1} \cdots a_{n} b_{n} f_{\alpha_{n}} \notin I$ but $a_{1} \cdots a_{n+1} b_{n} f_{\alpha_{n}} \in I$. Thus

$$
I: a_{1} a_{2} \cdots a_{n} \subset I: a_{1} a_{2} \cdots a_{n+1} \quad \forall n \in \mathbb{N}
$$

this contradicts $I$ being an abstract continuity ideal.

LEMMA 4.8. Let $J$ be a semiprime ideal in A. Let $a, b \in A$ be such that $J: a$ and $J: b$ are prime ideals. Then the following are equivalent.

(a) $J: a \subseteq J: b$.

(b) $a b \notin J$.

(c) $J: a=J: b$. 
PROOF. To show that (a) implies (b), we note that since $J$ is semiprime and $J: b$ is a proper ideal in $A$, we must have $b \notin J: b$. So $b \notin J: a$, and therefore $a b \notin J$.

To show that (b) implies (c), we observe that condition (b) implies that $a \notin J: b$. Let $f \in J: a$. Then $f a \in J \subseteq J: b$, and so $f \in J: b$ by the primeness of $J: b$. Thus $J: a \subseteq J: b$. Similarly, $J: b \subseteq J: a$.

REMARK 4.9. In the case where $I$ is semiprime, since $\mathfrak{P}$ is the set of prime ideals of the form $I: a$, the above lemma shows that, if $P=I: a \in \mathfrak{P}$, then $P$ is minimal in $\mathfrak{P}$, $a \in A \backslash P$, and $a \in Q$ whenever $Q \in \mathfrak{P} \backslash\{P\}$.

We can now state the main result of this section.

THEOREM 4.10. Suppose that I is an abstract continuity ideal of a commutative algebra A. Denote by $\mathfrak{P}_{0}$ the set of minimal ideals among the prime ideals of the form I : a for some a $\in$ A. Then:

(i) $\quad \sqrt{I}=\bigcap_{P \in \mathfrak{P}_{0}} P$;

(ii) $\mathfrak{P}_{0}$ is a relatively compact family of prime ideals.

PROOF. The first assertion follows from Lemmas 4.4 and 4.5. For the second, let $\left(P_{n}\right) \subseteq \mathfrak{P}_{0}$. We can assume that $P_{n}$ where $n \in \mathbb{N}$ are distinct. Set $J=\bigcap_{n=1}^{\infty} P_{n}$. By Lemma 4.6, there exists $a_{n} \in \bigcap_{i \neq n} P_{i} \backslash P_{n}$, and so $P_{n}=J: a_{n}$. Let $a \in A$ be such that $J: a$ is a prime ideal. We claim that $J: a \in\left\{P_{n}\right\}$. Indeed, we see that $a \notin J$, and thus $a \notin P_{n_{0}}$ for some $n_{0}$. So $a a_{n_{0}} \notin J$. By Lemma 4.8, we deduce that $J: a=P_{n_{0}}$. It then follows from Lemmas 4.7 and 4.3 (applied to $J$, with $\kappa$ being the first infinite cardinal $\left.\aleph_{0}\right)$ that $\left(P_{n}\right)$ must have a pseudofinite subsequence.

Corollary 4.11. Let $\theta: A \rightarrow B$ be a homomorphism from a Banach algebra $A$ into a commutative Banach algebra $B$. Then $\sqrt{\mathcal{I}(\theta)}$ is the intersection of a relatively compact family of prime ideals of the form $\mathcal{I}(\theta)$ : a for some a $\in A$.

REMARK 4.12. Since $\operatorname{ker} \theta \subseteq \mathcal{I}(\theta)$ and $A / \operatorname{ker} \theta$ is commutative, all the previous definitions and results still make sense in this case.

PROOF. It can be seen that, for all $a \in A$,

$$
(\mathcal{I}(\theta) / \operatorname{ker} \theta):(a+\operatorname{ker} \theta)=(\mathcal{I}(\theta): a) / \operatorname{ker} \theta .
$$

So by the stability lemma, we see that $\mathcal{I}(\theta) / \operatorname{ker} \theta$ is an abstract continuity ideal in $A / \operatorname{ker} \theta$. The rest follows from the theorem.

Corollary 4.13. Let $\theta: A \rightarrow B$ be an epimorphism from a Banach algebra A onto a commutative Banach algebra $B$. Then $\sqrt{\mathcal{I}(\theta)}$ is the intersection of a finite number of prime ideals of the form $\mathcal{I}(\theta):$ a for some $a \in A$ and there exists $k \in \mathbb{N}$ such that $\sqrt{\mathcal{I}(\theta)}=\left\{a \in A \mid a^{k} \in \mathcal{I}(\theta)\right\}$.

PROOF. This is proved in the same way as [15, Corollary 4.7]. 
LEMMA 4.14. Let I be an abstract continuity ideal of $\mathcal{C}_{0}(\Omega)$ for a locally compact space $\Omega$. Then I is either a semiprime ideal or the whole of $\mathcal{C}_{0}(\Omega)$.

PROOF. The proof is the same as the proof that the continuity ideal of a discontinuous homomorphism from $\mathcal{C}_{0}(\Omega)$ into a Banach algebra is semiprime ([6, 17], see also [3, Theorem 5.4.31]).

COROLlARY 4.15. Let $\Omega$ be a locally compact space.

(i) Let I be an abstract continuity ideal in $\mathcal{C}_{0}(\Omega)$. Denote by $\mathfrak{P}$ the set of prime ideals of the form $I: f$ for some $f \in \mathcal{C}_{0}(\Omega)$. Then:

(a) $I=\bigcap_{P \in \mathfrak{P}} P$;

(b) $\mathfrak{P}$ is a relatively compact family of prime ideals.

(ii) Conversely, let $\mathfrak{P}$ be a relatively compact family of prime ideals in $\mathcal{C}_{0}(\Omega)$. Then $\bigcap_{P \in \mathfrak{P}} P$ is an abstract continuity ideal in $\mathcal{C}_{0}(\Omega)$.

COROLlary 4.16. Let $\Omega$ be a locally compact space. Then each homomorphism from $\mathcal{C}_{0}(\Omega)$ into a Banach algebra is continuous on the intersection of a relatively compact family of prime ideals of the form $\mathcal{I}(\theta): f$ for some $f \in \mathcal{C}_{0}(\Omega)$.

\section{5. (Relatively) compact families of prime ideals in $\mathcal{C}_{0}(\Omega)$}

In this section, let $\Omega$ be a locally compact space, and let $\mathfrak{P}$ be a nonempty relatively compact family of prime ideals in $\mathcal{C}_{0}(\Omega)$. Denote by $\mathfrak{Q}$ the collection of all the ideals that are unions of countably many ideals in $\mathfrak{P}$. We call $\mathfrak{Q}$ the closure of $\mathfrak{P}$; we shall show that it is indeed the smallest compact family of prime ideals containing $\mathfrak{P}$.

Note that an ideal in $\mathfrak{Q}$ is automatically prime in $\mathcal{C}_{0}(\Omega)$, and that the union of each pseudofinite sequence of prime ideals in $\mathcal{C}_{0}(\Omega)$ is again a prime ideal in $\mathcal{C}_{0}(\Omega)$; this follows from the next lemma.

LEMMA 5.1. The union of finitely many prime ideals in $\mathcal{C}_{0}(\Omega)$ either is one of the given prime ideals or is not even a linear space. The union of countably many prime ideals in $\mathcal{C}_{0}(\Omega)$ is not equal to $\mathcal{C}_{0}(\Omega)$.

PROOF. We prove the second clause only; the proof of the first is similar. Let $\left(P_{n}\right.$ : $n \in \mathbb{N}$ ) be a sequence of prime ideals in $\mathcal{C}_{0}(\Omega)$. For all $n \in \mathbb{N}$, choose $f_{n} \in \mathcal{C}_{0}(\Omega) \backslash P_{n}$. We can assume that $0 \leq f_{n} \leq 2^{-n}$. Set $f=\sum_{n=1}^{\infty} f_{n}$. Then $f \in \mathcal{C}_{0}(\Omega)$ but $f \notin P_{n}$ since $f \geq f_{n}$ for all $n \in \mathbb{N}$.

LEMMA 5.2. Each chain in $\mathfrak{Q}$ is well ordered with respect to inclusion; that is, each nonempty chain in $\mathfrak{Q}$ has a smallest element.

Proof. Assume that the contrary holds. Then we can find an infinite chain in $\mathfrak{Q}$ : $\cdots \subset Q_{n} \subset \cdots \subset Q_{1}$. For all $n$, choose $P_{n} \in \mathfrak{P}$ such that $P_{n} \subseteq Q_{n}$ but $P_{n} \nsubseteq Q_{n+1}$. By the relative compactness of $\mathfrak{P}$, we can assume without loss of generality that $\left(P_{n}: n \in \mathbb{N}\right)$ is a pseudofinite sequence. Set $Q=\bigcup_{n=1}^{\infty} P_{n}$. Then $Q \in \mathfrak{Q}$, and for each $n \in \mathbb{N}$, either $Q_{n} \subseteq Q$ or $Q \subseteq Q_{n}$ (since both contain $P_{n}$ ). Since $P_{n-1} \nsubseteq Q_{n}$, we 
have $Q_{n} \subseteq Q$ for all $n \geq 2$. Choose $a \in Q_{2} \backslash Q_{3}$. Then $a \notin Q_{n}$, and so $a \notin P_{n}$ for all $n \geq 3$. However, $a \in Q=\bigcup_{n=1}^{\infty} P_{n}$. This contradicts the pseudofiniteness of $\left(P_{n}\right)$.

\section{LEMMA 5.3. $\mathfrak{Q}$ is compact.}

Proof. Let $\left(Q_{n}\right)$ be a sequence in $\mathfrak{Q}$. Let $P_{n} \in \mathfrak{P}$ such that $P_{n} \subseteq Q_{n}$. Since $\mathfrak{P}$ is relatively compact, we can assume without loss of generality that $\left(P_{n}\right)$ is pseudofinite; its union is denoted by $Q$. We see that either $Q_{n} \subseteq Q$ or $Q \subseteq Q_{n}$. If there are infinitely many $Q_{n}$ contained in $Q$, then those $Q_{n}$ form a pseudofinite sequence, following from the pseudofiniteness of $\left(P_{n}\right)$. On the other hand, if there are infinitely many $Q_{n}$ containing $Q$, then those $Q_{n}$ form a chain, and the previous lemma enables us to find a nondecreasing sequence of ideals. Thus $\mathfrak{Q}$ is relatively compact. The result then follows from the definition of $\mathfrak{Q}$.

\section{LEMMA 5.4. $\mathfrak{Q}$ is the set of unions of pseudofinite sequences of ideals in $\mathfrak{P}$.}

Proof. We only need to prove that each ideal $Q \in \mathfrak{Q}$ is the union of a pseudofinite sequence in $\mathfrak{P}$. For this purpose,we can suppose without loss of generality that $\mathfrak{P}$ is countable and that $Q$ is the union of $\mathfrak{P}$. It is obvious that, in this case, any chain in $\mathfrak{Q}$ is countable.

Case 1: $Q$ is the union of a chain of ideals in $\mathfrak{Q} \backslash\{Q\}$. By the countability and well-ordering of the chain, there exists a chain $Q_{1} \subset Q_{2} \subset \cdots \subset Q$ such that $Q=\bigcup_{n=1}^{\infty} Q_{n}$. For all $n$, choose $P_{n} \in \mathfrak{P}$ such that $P_{n} \subseteq Q_{n+1}$ but $P_{n} \nsubseteq Q_{n}$. Since $\mathfrak{P}$ is relatively compact, without loss of generality, we can assume that $\left(P_{n}\right)$ is pseudofinite; its union is denoted by $Q^{\prime}$. Then $Q^{\prime} \subseteq Q$, and for each $n \geq 2$, either $Q_{n} \subseteq Q^{\prime}$ or $Q^{\prime} \subseteq Q_{n}$ (since both contain $P_{n-1}$ ). Since $P_{n} \nsubseteq Q_{n}$, we must have $Q_{n} \subseteq Q^{\prime}$ for all $n \geq 2$. So $Q=Q^{\prime}$.

Case 2: $Q$ is not the union of any chain of ideals in $\mathfrak{Q} \backslash\{Q\}$. Then any $P \in$ $\mathfrak{Q} \backslash\{Q\}$ is contained in a maximal element of $\mathfrak{Q} \backslash\{Q\}$. Since $Q$ cannot be the union of any finite number of prime ideals properly contained in $Q$, either $\mathfrak{Q}=\{Q\}$, which implies that $Q \in \mathfrak{P}$, or there exist infinitely many maximal elements of $\mathfrak{Q} \backslash\{Q\}$. In the latter case, let $Q_{n}$, where $n \in \mathbb{N}$, be distinct maximal elements of $\mathfrak{Q} \backslash\{Q\}$. Choose $P_{n} \in \mathfrak{P}$ such that $P_{n} \subseteq Q_{n}$. By the relative compactness of $\mathfrak{P}$ and without loss of generality, we can assume that $\left(P_{n}: n \in \mathbb{N}\right)$ is a pseudofinite sequence. Set $Q^{\prime}=\bigcup_{n=1}^{\infty} P_{n}$. Then $Q^{\prime} \in \mathfrak{Q}$, and for each $n \in \mathbb{N}$, either $Q_{n} \subseteq Q^{\prime}$ or $Q^{\prime} \subseteq Q_{n}$. Assume towards a contradiction that $Q^{\prime} \neq Q$. The maximality of the ideal $Q_{n}$ in $\mathfrak{Q} \backslash\{Q\}$ implies that $Q^{\prime} \subseteq Q_{n}$ for all $n \in \mathbb{N}$. This implies that $\left\{Q_{n} \mid n \in \mathbb{N}\right\}$ form a chain, contradicting the maximality and distinction of the ideals $Q_{n}$. Thus $Q^{\prime}=Q$.

In both cases, $Q$ is the union of a pseudofinite sequence in $\mathfrak{P}$.

In summary, we have the following proposition.

PROPOSITION 5.5. The closure $\mathfrak{Q}$ of $\mathfrak{P}$ satisfies the following conditions.

(i) $\mathfrak{Q}$ is the set of unions of pseudofinite sequences of ideals in $\mathfrak{P}$.

(ii) $\mathfrak{Q}$ is compact. 
(iii) Every chain in $\mathfrak{Q}$ is well ordered with respect to the inclusion.

(iv) $\bigcap_{P \in \mathfrak{P}} P=\bigcap_{Q \in \mathfrak{Q}} Q$.

From (i), we see that $\mathfrak{Q}$ is the smallest compact family of prime ideals containing $\mathfrak{P}$. Property (iii) also shows that the intersection of $\mathfrak{P}$ is equal to the intersection of its minimal elements.

In the remainder of the section, we consider $\mathfrak{Q}$ to be any compact family of prime ideals in $\mathcal{C}_{0}(\Omega)$.

Lemma 5.6. Let $P$ be in $\mathfrak{Q}$. Then there exists $a \in A \backslash P$ such that $a \in Q$ for all $Q \in \mathfrak{Q}$ with $Q \nsubseteq P$.

PROOF. Assume that the contrary holds. As in Lemma 4.6, we can construct $\left(a_{n}\right) \subseteq A$ and $\left(Q_{n}\right) \subseteq \mathfrak{Q}$ such that $a_{1} \ldots a_{n} \notin Q_{n}$ but $a_{1} \ldots a_{n+1} \in Q_{n}$ for all $n \in \mathbb{N}$. By compactness, $\left(Q_{n}\right)$ has a pseudofinite subsequence $\left(Q_{n_{i}}\right)$. However, $a_{1} \ldots a_{n_{2}} \in Q_{n_{1}}$ but $a_{1} \ldots a_{n_{2}} \notin Q_{n_{i}}$ for all $i \geq 2$; this contradicts the pseudofiniteness.

We say that an ideal $Q$ is a roof of $\mathfrak{Q}$ if it is the union of the ideals in a maximal chain in $\mathfrak{Q}$. A roof must be either a prime ideal in $\mathcal{C}_{0}(\Omega)$ or $\mathcal{C}_{0}(\Omega)$ itself.

LEMMA 5.7. $\mathfrak{Q}$ has only finitely many roofs. Also, there are only finitely many maximal modular ideals in $\mathcal{C}_{0}(\Omega)$ such that each of them contains an ideal in $\mathfrak{Q}$.

PROOF. We shall prove that there are only finitely many disjoint maximal chains in $\mathfrak{Q}$; the lemma then follows. Assume to the contrary that $\mathfrak{C}_{n}$, where $n \in \mathbb{N}$, are disjoint maximal chains in $\mathfrak{Q}$. Pick $Q_{n} \in \mathfrak{C}_{n}$. Without loss of generality, we can suppose that ( $Q_{n}$ ) is pseudofinite; its union is denoted by $Q$. We see that $Q \in \mathfrak{Q}$, and since $Q \supseteq Q_{n}$ and $\mathfrak{C}_{n}$ is maximal, we have $Q \in \mathfrak{C}_{n}$ for all $n \in \mathbb{N}$, contradicting the disjointness of the chains $\mathfrak{C}_{n}$.

For a function $f \in \mathcal{C}_{0}(\Omega)$, we can define a new function $f / \sqrt{|f|}$, also in $\mathcal{C}_{0}(\Omega)$, as follows:

$$
\left(\frac{f}{\sqrt{|f|}}\right)(x)= \begin{cases}\frac{f(x)}{\sqrt{|f(x)|}} & \text { when } x \in \Omega \text { and } f(x) \neq 0 \\ 0 & \text { when } x \in \Omega \text { and } f(x)=0 .\end{cases}
$$

REMARK 5.8. If $P$ is a prime ideal in $\mathcal{C}_{0}(\Omega)$ and $f \in P$, then the function $f / \sqrt{|f|}$ defined above is also in $P$.

The following lemma and proposition are inspired by a suggestion of an anonymous referee of an initial version of [15].

LEMMA 5.9. Suppose that $\mathfrak{Q}$ is a compact family of prime ideals in $\mathcal{C}_{0}(\Omega)$ with a maximum element $Q$. Set $I=\bigcap_{P \in \mathfrak{Q}} P$. Let $a \in \mathcal{C}_{0}(\Omega) \backslash Q$ and let $b \in Q$. Then there exists $s \in Q$ such that as $-b \in I$.

PRoOF. It is standard that for each prime ideal $P \subseteq Q$, there exists $s \in Q$ such that $a s-b \in P$. 
Assume towards a contradiction that for all $s \in Q$, as $-b \notin I$. Initially, set $s_{1}=0, b_{1}=b-a s_{1}=b$, and $\mathfrak{Q}_{1}=\left\{P \in \mathfrak{Q} \mid b_{1} \notin P\right\}$. Suppose that we have already constructed $s_{n} \in Q, b_{n}=b-a s_{n}$, and $\mathfrak{Q}_{n}=\left\{P \in \mathfrak{Q} \mid b_{n} \notin P\right\}$ such that

$$
\mathfrak{Q}_{n} \subset \cdots \subset \mathfrak{Q}_{1} \text {. }
$$

Since $b_{n} \notin I$ by the assumption, $\mathfrak{Q}_{n} \neq \emptyset$. Choose $P_{n} \in \mathfrak{Q}_{n}$. We see that $b_{n} \in Q$, and so $b_{n} / \sqrt{\left|b_{n}\right|} \in Q$. Thus there exists $s^{\prime} \in Q$ such that $a s^{\prime}-\left(b_{n} / \sqrt{\left|b_{n}\right|}\right) \in P_{n}$. Now we set $s_{n+1}=s_{n}+s^{\prime} \sqrt{\left|b_{n}\right|}$,

$$
b_{n+1}=b-a s_{n+1}=\left(\frac{b_{n}}{\sqrt{\left|b_{n}\right|}}-a s^{\prime}\right) \sqrt{\left|b_{n}\right|},
$$

and $\mathfrak{Q}_{n+1}=\left\{P \in \mathfrak{Q} \mid b_{n+1} \notin P\right\}$. We see that $\mathfrak{Q}_{n+1} \subset \mathfrak{Q}_{n} ; P_{n} \in \mathfrak{Q}_{n} \backslash \mathfrak{Q}_{n+1}$. Thus the construction can be continued inductively.

In particular, when $m, n \in \mathbb{N}$, we have $b_{m} \in P_{n}$ if $m>n$, but $b_{m} \notin P_{n}$ if $m \leq n$. The compactness implies that there exists a pseudofinite subsequence $\left(P_{n_{i}}\right)$. However, this contradicts the fact that $b_{n_{2}} \in P_{n_{1}}$, but $b_{n_{2}} \notin P_{n_{i}}$ for all $i \geq 2$.

PROPOSITION 5.10. Suppose that $\Omega$ is noncompact and $\mathfrak{Q}$ is a compact family of nonmodular prime ideals in $\mathcal{C}_{0}(\Omega)$ with a maximum element $Q$. Set $I=\bigcap_{P \in \mathfrak{Q}} P$. Let $P \in \mathfrak{Q}$, and let $A$ be a subalgebra of $\mathcal{C}_{0}(\Omega)$. Suppose that $\mathcal{C}_{0}(\Omega)=A+P$ and $A \cap P$ is the intersection of a subfamily of $\mathfrak{Q}$. Let $B$ be a subalgebra of $A$ such that $B$ is maximal with respect to the property that $B \cap Q \subseteq I$. Then $\mathcal{C}_{0}(\Omega)=B+Q$.

PROOF. When $a-b \in I$ we also write $a=b \bmod I$.

Note that $I \subseteq A \cap P$. By maximality of $B$ we see that $I \subseteq B$, so indeed $B \cap Q=I$. We can also check, using the maximality of $B$ again, that $B \neq I$. Moreover, $I$ is nonmodular in $B$; for otherwise, $Q$ would be modular in $B+Q$ (because $B / I \cong$ $(B+Q) / Q)$, and from the primeness of $Q$ we could deduce that $Q$ is modular in $\mathcal{C}_{0}(\Omega)$, which would be a contradiction. Thus $I$ is indeed a nonmodular prime ideal in $B$, and hence, a prime ideal in $B^{\#}$.

We claim that for each $a \in A \backslash Q$ and each $b \in A \cap Q$, there exists $s \in A \cap Q$ such that $a s-b \in I$. Indeed, by the previous lemma, there exists $s \in Q$ such that $a s-b \in I$. Write $s=c+p$ where $c \in A$ and $p \in P$. Then $a p+(a c-b) \in I \subseteq A$ implies that $a p \in A \cap P$. Since $a \notin Q$ and $A \cap P$ is the intersection of a family of prime ideals contained in $Q$, we must have $p \in A \cap P$. Thus $s=c+p \in A \cap Q$.

We shall prove that $A=B+(A \cap Q)$; the proposition then follows. Indeed, assume towards a contradiction that $B+(A \cap Q) \subset A$. Let $a \in A$ such that $a \notin$ $B+Q$. By the maximality of $B$, there exists a polynomial $q(X)$ in $B^{\#}[X] \backslash I[X]$ such that $q(a) \in Q$. Let $q(X)$ be such a polynomial of smallest degree. By multiplying with some element in $B \backslash I$, we can further suppose that the coefficients of $q(X)$ are in $B$. Then we see that $q^{\prime}(a) \in A \backslash Q$, where $q^{\prime}(X)$ is the formal derivative. Let $s \in \mathcal{C}_{0}(\Omega)$. Then

$$
q\left(a+q^{\prime}(a) s\right)=q(a)+q^{\prime}(a)^{2} s+\cdots+q^{\prime}(a)^{n} q^{(n)}(a) \frac{s^{n}}{n !}
$$


where $q^{(k)}(X)$ is the formal $k$ th derivative of $q(X)$. By the claim, there exists $d \in A \cap Q$ such that

$$
q(a)=q^{\prime}(a)^{2} d \bmod I .
$$

So for all $s \in \mathcal{C}_{0}(\Omega)$,

$$
q\left(a+q^{\prime}(a) s\right)=q^{\prime}(a)^{2}\left(d+s+\cdots+q^{\prime}(a)^{n-2} q^{(n)}(a) \frac{s^{n}}{n !}\right) \bmod I .
$$

Since $(A /(A \cap P)) \cong \mathcal{C}_{0}(\Omega) / P$ is radical and $\left(\mathcal{C}_{0}(\Omega) / P\right)^{\#}$ is Henselian, there exists $s \in A$ such that

$$
d+s+\cdots+q^{\prime}(a)^{n-2} q^{(n)}(a) \frac{s^{n}}{n !} \in A \cap P \subseteq Q
$$

(by [3, Theorem 2.4.30 and Proposition 1.6.3]). It follows that $s \in Q$ (so $s \in A \cap Q$ ) and

$$
q\left(a+q^{\prime}(a) s\right) \in A \cap P .
$$

If we set $b=a+q^{\prime}(a) s$, then $b \in A \backslash(B+Q), q(b) \in P$, and $q(X)$ is a polynomial of smallest degree among all the polynomials in $B^{\#}[X] \backslash I[X]$ such that $q(b) \in Q$.

Hence, without loss of generality, we can assume from the start that $q(a) \in P$. In this case, $d \in P$ by (5.1), so $d \in A \cap P$. Again, since $\mathcal{C}_{0}(\Omega) / I$ is radical and $\mathcal{C}_{0}(\Omega)^{\#} / I$ is Henselian, there exists $t \in \mathcal{C}_{0}(\Omega)$ such that

$$
d+t+\cdots+q^{\prime}(a)^{n-2} q^{(n)}(a) \frac{t^{n}}{n !} \in I \subseteq A \cap P .
$$

Now $A \cap P$ is an ideal in $\mathcal{C}_{0}(\Omega)$, and so $t \in A \cap P$. Set $c=a+q^{\prime}(a) t$. Then $c \in A \backslash(B+Q), q(c) \in I$, and $q(X)$ is a polynomial of smallest degree among all the polynomials in $B^{\#}[X] \backslash I[X]$ such that $q(c) \in Q$. The maximality of $B$ implies that there exists a polynomial $p(X)$ with coefficients in $B^{\#}$ such that $p(c) \in Q \backslash I$. We see that there exist $u \in B \backslash I$ and a polynomial $h(X)$ with coefficients in $B^{\#}$ such that $u p(X)=q(X) h(X) \bmod I$; this is possible since $I$ is prime in $B^{\#}$. Then $u p(c)=q(c) h(c)=0 \bmod I$. Since $u \notin Q$ (otherwise, $u \in B \cap Q=I$ ) and $I$ is the intersection of some prime ideals contained in $Q$, we deduce that $p(c) \in I$; a contradiction.

A special case of the previous proposition is when $A=\mathcal{C}_{0}(\Omega)$.

\section{Homomorphisms from $\mathcal{C}_{0}(\Omega)$}

In this section, we shall show the connection between continuity ideals as well as the kernels of homomorphisms from $\mathcal{C}_{0}(\Omega)$ into Banach algebras and intersections of (relatively) compact families of prime ideals. One direction is an immediate consequence of the results in Section 4, so most of this section concerns the converse.

We shall need some basic complex algebraic-geometry results; we are indebted to [14] for these. For a set $S \subseteq \mathbb{C}\left[Z_{1}, Z_{2}, \ldots, Z_{n}\right]$, denote by $\mathcal{V}(S)$ the variety (that 
is, common zero set) of $S$ in $\mathbb{C}^{n}$. For each prime ideal $Q$ in $\mathbb{C}\left[Z_{1}, \ldots, Z_{n}\right]$, the variety $\mathcal{V}(Q)$ is irreducible. The topology considered on complex spaces will be the Euclidean topology. We shall need the fact that, for each irreducible variety $V$ and each variety $W$ not containing $V$, the set $V \backslash W$ is dense and (relatively) open in $V$ [14, Ch. IV, Theorem 2.11].

Notation. For clarity, we shall use $X_{i}, Y_{j}$ for variables, $x_{i}, y_{j}$ for complex numbers, and $a_{i}, b_{j}$ for elements of an algebra. When there is no ambiguity, we shall use boldface characters to denote tuples of elements of the same type; for example, we set

$$
\boldsymbol{X}=\left(X_{1}, X_{2}, \ldots, X_{m}\right) \quad \text { or } \quad \boldsymbol{y}=\left(y_{1}, \ldots, y_{n}\right) \text {. }
$$

In the case where $\boldsymbol{X}=\left(X_{1}, \ldots, X_{m}\right)$, we also denote by $\mathbb{C}_{\boldsymbol{X}}$ the corresponding space $\mathbb{C}^{m}$.

Lemma 6.1. Let $m, n \in \mathbb{N}$, and let $Q$ be a prime ideal in $\mathbb{C}[\boldsymbol{X}, \boldsymbol{Y}]$, where $\boldsymbol{X}=$ $\left(X_{1}, \ldots, X_{m}\right)$ and $\boldsymbol{Y}=\left(Y_{1}, \ldots, Y_{n}\right)$. Consider $Q_{X}=Q \cap \mathbb{C}[X]$ as a prime ideal in $\mathbb{C}[X]$. Let $V$ be the variety of $Q$, and let $V_{X}$ be the variety of $Q_{X}$. Let $\pi$ be the natural projection $\mathbb{C}_{X, Y} \rightarrow \mathbb{C}_{X}$. Then $\pi: V \rightarrow V_{X}$ and there exists a dense open subset $U$ of $V$ such that $\pi: U \rightarrow V_{X}$ is an open map.

ProOf. Obviously, $\pi$ maps $V$ to $V_{\boldsymbol{X}}$. Without loss of generality, take a transcendental basis $\left(X_{1}, \ldots, X_{k}\right)$ for $\mathbb{C}[X]$ modulo $Q_{X}$. We consider $\mathbb{C}^{k}=\mathbb{C}_{X_{1}, \ldots, X_{k}}$. Denote by $\pi_{1}$ the natural projection $\mathbb{C}_{\boldsymbol{X}, \boldsymbol{Y}} \rightarrow \mathbb{C}^{k}$, and by $\pi_{2}$ the natural projection $\mathbb{C}_{\boldsymbol{X}} \rightarrow \mathbb{C}^{k}$.

By [15, Lemma 6.3], there exist dense open subsets $U$ of $V$ and $U_{\boldsymbol{X}}$ of $V_{\boldsymbol{X}}$, such that $\pi_{1}: U \rightarrow \mathbb{C}^{k}$ and $\pi_{2}: U_{X} \rightarrow \mathbb{C}^{k}$ are open maps. Inspecting the proof of [15, Lemma 6.3], we see that $U_{\boldsymbol{X}}$ can be chosen as $V_{\boldsymbol{X}} \backslash V_{0}$, where $V_{0}$ is a proper subvariety of $V_{\boldsymbol{X}}$, and that $\pi_{2}$ is a local homeomorphism from $U_{\boldsymbol{X}}$ onto an open subset of $\mathbb{C}^{k}$. Since $V_{0}$ has dimension at most $k-1$ [14, Ch. IV], by shrinking $U_{\boldsymbol{X}}$ if necessary, we can further require that

$$
\pi_{2}\left(U_{X}\right) \cap \pi_{2}\left(V_{X} \backslash U_{X}\right)=\emptyset .
$$

Let $W=\pi_{1}(U) \cap \pi_{2}\left(U_{\boldsymbol{X}}\right)$. Then $W$ is an open set in $\mathbb{C}^{k}$. It can be seen that $W$ is dense in $\pi_{1}(U)$. Set

$$
U^{\prime}=U \cap \pi_{1}^{-1}(W) .
$$

Then $U^{\prime}$ is a dense open subset of $U$, by the openness of $\pi_{1}: U \rightarrow \mathbb{C}^{k}$, and hence a dense open subset of $V$.

We claim that $\pi: U^{\prime} \rightarrow V_{\boldsymbol{X}}$ is an open map. Indeed, let $(\boldsymbol{x}, \boldsymbol{y}) \in U^{\prime}$ be arbitrary. Then $\left(x_{1}, \ldots, x_{k}\right) \in W$, and so $\boldsymbol{x} \in U_{\boldsymbol{X}}$ by (6.1). Choose $\Delta_{\boldsymbol{X}}$ to be a neighbourhood of $\boldsymbol{x}$ in $U_{\boldsymbol{X}}$ such that $\pi_{2}$ is a homeomorphism from $\Delta_{\boldsymbol{X}}$ onto an open subset of $\mathbb{C}^{k}$. Let $\Delta$ be any neighbourhood of $(\boldsymbol{x}, \boldsymbol{y})$ in $U^{\prime}$ contained in $\pi^{-1}\left(\Delta_{X}\right) \cap U^{\prime}$. Then we see that $\pi(\Delta) \subseteq \Delta_{X}$ and $\pi_{2}(\pi(\Delta))=\pi_{1}(\Delta)$, which is open in $\mathbb{C}^{k}$ by the openness of $\pi_{1}$ on $U^{\prime}$. It follows that $\pi(\Delta)$ is open in $\Delta_{X}$ and thus in $V_{X}$. 
Proposition 6.2. Let $A=\mathcal{C}_{0}(\Omega)$ for a locally compact space $\Omega$, and let $\mathfrak{Q}$ be a nonempty compact family of nonmodular prime ideals in A. Suppose that every chain in $\mathfrak{Q}$ is countable. Then there exist a cardinal $\kappa$, a free ultrafilter $\mathcal{U}$ on $\kappa$, and a homomorphism $\theta_{P}: A \rightarrow\left(\mathbb{C}^{\mathcal{K}} / \mathcal{U}\right)^{\circ}$ for each $P \in \mathfrak{Q}$, such that:

(a) $\operatorname{ker} \theta_{P}=P$ for all $P \in \mathfrak{Q}$; and

(b) the $\operatorname{set}\left\{\theta_{P}(a) \mid P \in \mathfrak{Q}\right\}$ is finite for all $a \in A$.

Note that $\Omega$ must be noncompact and $A$ must be nonunital. Since each $P \in \mathfrak{Q}$ is a nonmodular prime ideal in $A$, it is a prime ideal in $A^{\#}$. For all $Q \in \mathfrak{Q}$, set

$$
\mathfrak{Q}_{Q}=\{P \in \mathfrak{Q} \mid P \subseteq Q\},
$$

and set $I_{Q}=\bigcap_{P \in \mathfrak{Q}_{Q}} P$. We begin the proof of Proposition 6.2 with some lemmas.

Lemma 6.3. Suppose that $Q_{*}, Q^{*} \in \mathfrak{Q}$ and $A_{*}$ and $A^{*}$ are subalgebras of $A$, such that $Q_{*} \subseteq Q^{*}$ and $A_{*} \supseteq A^{*}$. Suppose further that $A^{*} \cap Q^{*} \subseteq I_{Q^{*}}, A_{*} \cap Q_{*}=I_{Q_{*}}$, and $A_{*}+Q_{*}=A$. Let $\mathfrak{C}$ be a chain in $\mathfrak{Q}_{Q^{*}}$ where each ideal in $\mathfrak{C}$ contains $Q_{*}$. Then we can find subalgebras $A_{Q} \subseteq A$ where $Q \in \mathfrak{C}$ satisfying the following conditions.

(i) $A_{Q} \cap Q=I_{Q}$ and $A=A_{Q}+Q$ for all $Q \in \mathfrak{C}$.

(ii) Whenever $Q_{1}, Q_{2} \in \mathfrak{C}$ and $Q_{1} \subseteq Q_{2}$, then $A_{*} \supseteq A_{Q_{1}} \supseteq A_{Q_{2}} \supseteq A^{*}$.

PROOF. Assume towards a contradiction that the lemma fails for some instance of $Q_{*}, Q^{*}, A_{*}, A^{*}$, and $\mathfrak{C}$; we can further require that o(C) is smallest among such instances (note that $\mathfrak{C}$ is well ordered by Proposition 5.5(iii)).

Now $A_{*} \cap Q_{*}=\bigcap_{P \in \mathfrak{Q}_{Q_{*}}} P$ is the intersection of a subfamily of $\mathfrak{Q}_{Q}$, and

$$
A^{*} \cap Q \subseteq A^{*} \cap Q^{*} \subseteq I_{Q^{*}} \subseteq I_{Q},
$$

for all $Q \in \mathfrak{C}$. Thus we are in a situation where Proposition 5.10 applies.

Proposition 5.10 implies that $\mathfrak{C}$ is infinite; otherwise, we can use finite induction to construct $\left(A_{Q}: Q \in \mathfrak{C}\right.$ ). If $\mathfrak{C}$ is order isomorphic to $\omega$, the first infinite ordinal, say

$$
\mathfrak{C}=\left\{Q_{1} \subset Q_{2} \subset \cdots\right\}
$$

then Proposition 5.10 also enables us to construct $\left(A_{Q_{n}}\right)$ inductively.

In general, there exists a sequence $\left(Q_{n}\right)$ in $\mathfrak{C}$ converging to $Q_{\infty}=\bigcup_{Q \in \mathfrak{C}} Q$ in the order topology (this is where our proof needs the countability condition in Proposition 6.2). If $Q_{\infty} \in \mathfrak{C}$, we can construct $A_{Q_{\infty}}$ by Proposition 5.10. As in the previous paragraph, we can then find $A_{Q_{n}}$ for all $n \in \mathbb{N}$ satisfying both conditions (i) and (ii). The ideals $Q_{n}$ divide $\mathfrak{C} \backslash\left\{Q_{\infty}\right\}$ into chains that are order isomorphic

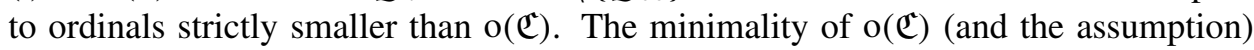
then imply that we can extend the present collection $\left\{A_{Q}\right\}$ to all $Q \in \mathfrak{C}$ that satisfy conditions (i) and (ii).

Thus we have a contradiction in either case. 
LEMma 6.4. We can find subalgebras $A_{Q} \subseteq$ A for all $Q \in \mathfrak{Q}$ satisfying the following conditions.

(i) $A_{Q} \cap Q=I_{Q}$ and $A=A_{Q}+Q$ for all $Q \in \mathfrak{Q}$.

(ii) $A_{Q_{1}} \supseteq A_{Q_{2}}$ whenever $Q_{1}, Q_{2} \in \mathfrak{Q}$ and $Q_{1} \subseteq Q_{2}$.

PROOF. This follows from the previous lemma, Zorn's lemma, and the fact that all prime ideals containing a given prime ideal in $A=\mathcal{C}_{0}(\Omega)$ form a chain.

Let $\kappa$ be the set of all tuples of the form $\left(\delta ; \mathfrak{G} ; a_{1}, \ldots, a_{m}\right)$, where $\delta>0, \mathfrak{G}$ is a nonempty finite subset of $\mathfrak{Q}$, and $\left(a_{1}, \ldots, a_{m}\right)$ is a nonempty finite sequence of distinct elements in $A$. Define a partial order $\prec$ on $\kappa$ by setting

$$
\left(\delta ; \mathfrak{G} ; a_{1}, a_{2}, \ldots, a_{m}\right) \prec\left(\delta^{\prime} ; \mathfrak{G}^{\prime} ; a_{1}^{\prime}, a_{2}^{\prime}, \ldots, a_{m^{\prime}}^{\prime}\right)
$$

if $\delta>\delta^{\prime}, \mathfrak{G} \subseteq \mathfrak{G}^{\prime},\left\{a_{1}, \ldots, a_{m}\right\}$ is a subset of $\left\{a_{1}^{\prime}, a_{2}^{\prime}, \ldots, a_{m^{\prime}}^{\prime}\right\}$. Then $(\kappa, \prec)$ is a net. Fix an ultrafilter $\mathcal{U}$ on $\kappa$ majorizing this net.

LeMma 6.5. Let $\left(A_{Q}: Q \in \mathfrak{Q}\right)$ be as in Lemma 4.6. Let $w=\left(\delta ; \mathfrak{G} ; a_{1}, \ldots, a_{m}\right) \in \kappa$. Then, for each $P \in \mathfrak{G}$, we can find a tuple

$$
\tau_{P}(w)=\boldsymbol{x}^{(P)}=\left(x_{1}^{(P)}, \ldots, x_{m}^{(P)}\right) \text { in } \mathbb{C}^{m}
$$

satisfying all the following conditions.

(i) $p\left(x_{1}^{(P)}, \ldots, x_{m}^{(P)}\right)=0$ for all $p \in \mathbb{C}\left[X_{1}, \ldots, X_{m}\right]$ with $p\left(a_{1}, \ldots, a_{m}\right) \in P$.

(ii) $x_{k}^{(P)} \neq 0$ for all $1 \leq k \leq m$ with $a_{k} \notin P$.

(iii) $\left|x_{k}^{(P)}\right| \leq \delta$ for all $1 \leq k \leq m$.

(iv) $x_{k}^{(P)}=x_{k}^{(Q)}$ for all $1 \leq k \leq m$ and all $P, Q \in \mathfrak{G}$ such that $P \subseteq Q$ and $a_{k} \in A_{Q}$.

Proof. Set $\boldsymbol{X}=\left(X_{1}, \ldots, X_{m}\right)$. Further, set $\boldsymbol{a}^{P}=\left(a_{1}, \ldots, a_{m}\right) \cap A_{P}$ and $\boldsymbol{X}^{P}=$ $\left(X_{i}: a_{i} \in A_{P}\right)$ for all $P \in \mathfrak{G}$. Without loss of generality, we can assume that none of these is empty. Note that, if $P, Q \in \mathfrak{G}$ and $P \subseteq Q$, then $\boldsymbol{a}^{Q} \subseteq \boldsymbol{a}^{P}$ and $\boldsymbol{X}^{Q} \subseteq \boldsymbol{X}^{P}$.

Denote by $\pi_{P}$ the projection from $\mathbb{C}_{X}$ onto $\mathbb{C}_{X^{P}}$, and by $\pi_{P Q}$ the projection from $\mathbb{C}_{\boldsymbol{X}^{P}}$ onto $\mathbb{C}_{\boldsymbol{X}^{Q}}$ whenever $P, Q \in \mathfrak{G}$ and $P \subseteq Q$. We also conveniently consider $\mathbb{C}_{\boldsymbol{X}^{P}}$ as a subspace of $\mathbb{C}_{\boldsymbol{X}}$.

For each $Q \in \mathfrak{G}$, define

$$
\begin{aligned}
& \widetilde{Q}=\{p \in \mathbb{C}[\boldsymbol{X}] \mid p(\boldsymbol{a}) \in Q\}, \\
& \widehat{Q}=\left\{p \in \mathbb{C}\left[\boldsymbol{X}^{Q}\right] \mid p\left(\boldsymbol{a}^{Q}\right) \in Q\right\} .
\end{aligned}
$$

We see that if $P, Q \in \mathfrak{G}$ and $P \subseteq Q$, then $\widetilde{P} \subseteq \widetilde{Q}$ are prime ideals in $\mathbb{C}[\boldsymbol{X}]$, and $\widehat{Q}=\widetilde{Q} \cap \mathbb{C}\left[\boldsymbol{X}^{Q}\right]$ is a prime ideal in $\mathbb{C}\left[\boldsymbol{X}^{Q}\right]$. Since $A_{Q} \cap Q=A_{Q} \cap P$ in this case,

$$
\widehat{Q}=\left\{p \in \mathbb{C}\left[\boldsymbol{X}^{Q}\right] \mid p\left(\boldsymbol{a}^{Q}\right) \in P\right\}=\widetilde{P} \cap \mathbb{C}\left[\boldsymbol{X}^{Q}\right] ;
$$

and thus $\widehat{Q}=\mathbb{C}\left[\boldsymbol{X}^{Q}\right] \cap \widehat{P}$. 
It follows from Lemma 6.1 that there exist, for all $Q \in \mathfrak{G}$, dense open subsets $U_{Q}$ of $\mathcal{V}(\widetilde{Q})$ and $W_{Q}$ of $\mathcal{V}(\widehat{Q})$, such that:

(1) $\pi_{Q}: U_{Q} \rightarrow W_{Q}$ is an open map;

(2) $\pi_{P Q}: W_{P} \rightarrow W_{Q}$ is an open map whenever $P, Q \in \mathfrak{G}$ and $P \subseteq Q$.

(There are only finitely many varieties here.)

For each $Q \in \mathfrak{G}$, set

$$
V_{Q}=\bigcup_{\substack{1 \leq r \leq m \\ a_{r} \notin Q}}\left\{\boldsymbol{x}=\left(x_{1}, \ldots, x_{m}\right) \mid x_{r}=0\right\} .
$$

Then $V_{Q}$ is a variety that does not contain $\mathcal{V}(\widetilde{Q})$, and so $\mathcal{V}(\widetilde{Q}) \backslash V_{Q}$ is also a dense open subset of $\mathcal{V}(\widetilde{Q})$ because $\mathcal{V}(\widetilde{Q})$ is irreducible. Therefore, $U_{Q} \backslash V_{Q}$ is again a dense open subset of $\mathcal{V}(\widetilde{Q})$.

Set

$$
\Delta=\left\{\boldsymbol{x}=\left(x_{1}, \ldots, x_{m}\right):\left|x_{r}\right|<\delta \text { when } 1 \leq r \leq m\right\} .
$$

Finally, set $U_{Q}^{\prime}=\left(U_{Q} \backslash V_{Q}\right) \cap \Delta$ and $W_{Q}^{\prime}=W_{Q} \cap \Delta$. Note that the origin $\mathbf{0}$ is in $\mathcal{V}(\widetilde{Q})$ and $\mathcal{V}(\widehat{Q})$ for all $Q \in \mathfrak{G}$. So $U_{Q}^{\prime}$ and $W_{Q}^{\prime}$ are nonempty open subsets of $\mathcal{V}(\widetilde{Q})$ and $\mathcal{V}(\widehat{Q})$ respectively.

Conditions (i), (ii), and (iii) will be satisfied as long as we choose $\boldsymbol{x}^{(Q)} \in U_{Q}^{\prime}$ for all $Q \in \mathfrak{G}$. We now turn our attention to condition (iv).

Fix $P, Q \in \mathfrak{G}$ such that $P \subseteq Q$. Now $U_{P} \backslash V_{P}$ is dense in $\mathcal{V}(\widetilde{P})$ and $U_{Q}^{\prime} \subseteq \mathcal{V}(\widetilde{Q}) \subseteq$ $\mathcal{V}(\widetilde{P})$, so $\pi_{Q}\left(U_{P}^{\prime}\right) \cap \pi_{Q}\left(U_{Q}^{\prime}\right)$ is dense (and open) in $\pi_{Q}\left(U_{Q}^{\prime}\right)$ (which is open in $W_{Q}$ ). Note that $\pi_{Q}\left(U_{P}^{\prime}\right)=\pi_{P Q}\left[\pi_{P}\left(U_{P}^{\prime}\right)\right]$. In fact, we see that $\pi_{P Q}(D) \cap \pi_{Q}\left(U_{Q}^{\prime}\right)$ is dense and open in $\pi_{Q}\left(U_{Q}^{\prime}\right)$ for every dense open subset $D$ of $\pi_{P}\left(U_{P}^{\prime}\right)$.

Thus we can define a dense open subset $D_{Q}$ of $\pi_{Q}\left(U_{Q}^{\prime}\right.$ ) (in $\mathbb{C}_{X^{Q}}$ ) for each $Q \in \mathfrak{G}$ as follows. For all $P$ minimal in $\mathfrak{G}$, set $D_{P}=\pi_{P}\left(U_{P}^{\prime}\right)$. Then define inductively

$$
D_{Q}=\bigcap_{\substack{P \in \mathfrak{G} \\ P \subset Q}} \pi_{P Q}\left(D_{P}\right) \cap \pi_{Q}\left(U_{Q}^{\prime}\right) .
$$

The nonemptiness of the sets $D_{P}$ then allows us to pick tuples $\alpha^{Q} \in D_{Q}$ for all $Q \in \mathfrak{G}$, such that $\boldsymbol{\alpha}^{Q}=\pi_{P Q}\left(\boldsymbol{\alpha}^{P}\right)$ when $P, Q \in \mathfrak{G}$ and $P \subseteq Q$. (This can be done inductively, starting from the maximal elements in $\mathfrak{G}$; note that $\mathfrak{G}$ is finite and that the prime ideals containing a given prime ideal form a chain.) Finally, for all $Q \in \mathfrak{G}$, choose $\boldsymbol{x}^{(Q)} \in U_{Q}^{\prime}$ (in $\mathbb{C}^{m}=\mathbb{C}_{\boldsymbol{X}}$ ) such that $\pi_{Q}\left(\boldsymbol{x}^{(Q)}\right)=\boldsymbol{\alpha}^{Q}$. It can be checked that these are the desired tuples.

Proof of Proposition 6.2. We retain the notation of the previous lemmas. Define $\xi_{P}: A \rightarrow \mathbb{C}^{\kappa}$ for all $P \in \mathfrak{Q}$ as follows. For each $a \in A$ and $w=\left(\delta ; \mathfrak{G} ; a_{1}, \ldots, a_{m}\right) \in \kappa$, if $P \in \mathfrak{G}$ and if $a$ is in $\left(a_{1}, \ldots, a_{m}\right)$, say $a=a_{k}$ (there is at most one such $k$ ), 
then set $\xi_{P}(a)(w)=\tau_{P}(w)(k)$; otherwise, set $\xi_{P}(a)(w)=0$. Define $\theta_{P}(a)$ to be the equivalence class in $\mathbb{C}^{\kappa} / \mathcal{U}$ containing $\xi_{P}(a)$.

It follows from Lemma 6.5 that $\theta_{P}$ is an algebra homomorphism from $A$ into $\left(\mathbb{C}^{\kappa} / \mathcal{U}\right)^{\circ}$ with $\operatorname{ker} \theta_{P}=P$, for all $P \in \mathfrak{Q}$. Moreover, whenever $P, Q \in \mathfrak{G}, P \subseteq Q$ and $a \in A_{Q}$, we have $\theta_{P}(a)=\theta_{Q}(a)$.

Let $\left(P_{n}\right)$ be any pseudofinite sequence in $\mathfrak{Q}$, and let $Q=\bigcup_{n=1}^{\infty} P_{n}$. Let $a \in A$. Write $a=b+x$, where $b \in A_{Q}$ and $x \in Q$. Then $x \in P_{n}$ for all $n>n_{0}$ for some $n_{0} \in \mathbb{N}$. Therefore $\theta_{P_{n}}(a)=\theta_{P_{n}}(b)=\theta_{Q}(b)$ for all $n>n_{0}$. Thus the sequence $\left(\theta_{P_{n}}(a)\right)$ is eventually constant.

Finally, assume towards a contradiction that the set $\left\{\theta_{P}(a) \mid P \in \mathfrak{Q}\right\}$ is infinite for some $a \in A$. Let $\left(P_{n}\right)$ be a sequence in $\mathfrak{Q}$ such that all $\theta_{P_{n}}(a)$ are distinct. By the compactness of $\mathfrak{Q}$, we can assume that $\left(P_{n}\right)$ is pseudofinite. However, the previous paragraph shows that the set $\left\{\theta_{P_{n}}(a) \mid n \in \mathbb{N}\right\}$ is finite. This contradiction concludes the proof of Proposition 6.2.

REMARK 6.6. The idea of mapping into an ultrapower in the above proposition generalizes the approach in [5] of the theorem of Esterle [9] on embedding integral domains into radical Banach algebras.

We are now ready to prove our main results.

THEOREM 6.7. Suppose that $\Omega$ is a locally compact space.

(i) Let $\theta$ be a homomorphism from $\mathcal{C}_{0}(\Omega)$ into a radical Banach algebra $R$. Then $\operatorname{ker} \theta$ is the intersection of a (relatively) compact family of nonmodular prime ideals in $\mathcal{C}_{0}(\Omega)$.

(ii) $(\mathrm{CH})$ Let $\mathrm{I}$ be the intersection of a relatively compact family $\mathfrak{P}$ of nonmodular prime ideals in $\mathcal{C}_{0}(\Omega)$ such that every chain in the closure of $\mathfrak{P}$ is countable. Suppose that

$$
\left|\mathcal{C}_{0}(\Omega) / I\right|=\mathfrak{c} .
$$

Then there exists a homomorphism $\theta$ from $\mathcal{C}_{0}(\Omega)$ into a radical Banach algebra such that $\operatorname{ker} \theta=I$.

PROOF. (i) Since $\theta$ maps into a radical algebra, we see that $\operatorname{ker} \theta: f$ is nonmodular for each $f \in \mathcal{C}_{0}(\Omega)$. Theorem 1.1 shows that $\operatorname{ker} \theta=\mathcal{I}(\theta)$, and so it is an abstract continuity ideal in $\mathcal{C}_{0}(\Omega)$. The result then follows from Corollary 4.15(i). Proposition 5.5 then allows us to pass from a relatively compact family of prime ideals to a compact family of prime ideals with the same intersection.

(ii) Let $\mathfrak{Q}$ be the closure of $\mathfrak{P}$. Then every chain in $\mathfrak{Q}$ is countable, every ideal in $\mathfrak{Q}$ is nonmodular, and $I=\bigcap_{P \in \mathfrak{Q}} P$.

Let $\theta_{P}: \mathcal{C}_{0}(\Omega) \rightarrow\left(\mathbb{C}^{\kappa} / \mathcal{U}\right)^{\circ}$, where $P \in \mathfrak{Q}$, be the homomorphisms defined in Proposition 6.2. Let $B$ be the subalgebra of $\left(\mathbb{C}^{\kappa} / \mathcal{U}\right)^{\circ}$ generated by all the images of $\theta_{P}$ for all $P \in \mathfrak{Q}$. Then $B$ is a nonunital integral domain. Also

$$
|B|=\left|\bigcup_{P \in \mathfrak{Q}} \theta_{P}\left(\mathcal{C}_{0}(\Omega)\right)\right|=\left|\bigcup_{a \in \mathcal{C}_{0}(\Omega)}\left\{\theta_{P}(a) \mid P \in \mathfrak{Q}\right\}\right|=\mathfrak{c}
$$


since, for each $b \in a+I$,

$$
\left\{\theta_{P}(b) \mid P \in \mathfrak{Q}\right\}=\left\{\theta_{P}(a) \mid P \in \mathfrak{Q}\right\},
$$

which is finite. Thus [9] there exists an embedding $\psi: B \rightarrow R_{0}$ where $R_{0}$ is any universal radical Banach algebra. Then the map

$$
\theta: \mathcal{C}_{0}(\Omega) \rightarrow \prod_{P \in \mathfrak{Q}} R_{0}, \quad a \mapsto\left(\left(\psi \circ \theta_{P}\right)(a): P \in \mathfrak{Q}\right)
$$

is a homomorphism with kernel $\bigcap_{P \in \mathfrak{Q}} P=I$. It follows from Proposition 6.2 that the image of $\theta$ is contained in the radical of $\ell^{\infty}\left(\mathfrak{Q}, R_{0}\right)$.

THEOREM 6.8. Suppose that $\Omega$ is a locally compact space.

(i) Let $\theta$ be a homomorphism from $\mathcal{C}_{0}(\Omega)$ into a Banach algebra B. Then $\mathcal{I}(\theta)$ is the intersection of a (relatively) compact family of prime ideals in $\mathcal{C}_{0}(\Omega)$.

(ii) $(\mathrm{CH})$ Let $\mathrm{I}$ be the intersection of a relatively compact family $\mathfrak{P}$ of prime ideals in $\mathcal{C}_{0}(\Omega)$ such that every chain in the closure of $\mathfrak{P}$ is countable. Suppose that

$$
\left|\mathcal{C}_{0}(\Omega) / I\right|=\mathfrak{c} .
$$

Then there exists a homomorphism $\theta$ from $\mathcal{C}_{0}(\Omega)$ into a Banach algebra such that $\mathcal{I}(\theta)=I$.

Proof. (i) The continuity ideal $\mathcal{I}(\theta)$ is an abstract continuity ideal in $\mathcal{C}_{0}(\Omega)$. The result then follows from Corollary 4.15(i) and Proposition 5.5.

(ii) Denote by $\mathfrak{P}_{0}$ the set of nonmodular ideals in $\mathfrak{P}$. Let $\mathfrak{Q}^{\prime}$ be the closure of $\mathfrak{P} \backslash \mathfrak{P}_{0}$. By Lemma 5.7, $\mathfrak{Q}^{\prime}$ has only finitely many roofs. Denote by $Q_{1}, \ldots, Q_{n}$ the roofs of $\mathfrak{Q}^{\prime}$, and set $\mathfrak{P}_{i}=\left\{P \in \mathfrak{P} \backslash \mathfrak{P}_{0} \mid P \subseteq Q_{i}\right\}$.

Let $1 \leq k \leq n$. First, it is easy to see that $Q_{k}$ is a modular prime ideal. Pick a modular identity $u$ for $Q_{k}$, and pick $a \notin Q_{k}$. Then $a-a u \in Q_{k}$, and so, by Lemma 5.9, there exists $v \in Q_{k}$ such that $a-a u-a v \in \bigcap_{P \in \mathfrak{P}_{k}} P$. It follows easily that $u+v$ is a modular identity for $\bigcap_{P \in \mathfrak{P}_{k}} P$; denote it by $u_{k}$.

Theorem 6.7 shows that there exists a homomorphism $\theta_{0}$ from $\mathcal{C}_{0}(\Omega)$ into a radical Banach algebra $R_{0}$ such that $\operatorname{ker} \theta_{0}=\bigcap_{P \in \mathfrak{P}_{0}} P$. Similarly, when $1 \leq k \leq n$, there exists a homomorphism $\theta_{k}$ from $M_{k}$ into $R_{k}$ such that $\operatorname{ker} \theta_{k}=\bigcap_{P \in \mathfrak{P}_{k}} P$, where $M_{k}$ is the maximal modular ideal containing $Q_{k}$. We extend $\theta_{k}$ to a homomorphism from $\mathcal{C}_{0}(\Omega)$ into $R^{\#}$, setting $\theta_{k}\left(u_{k}\right)=\mathbf{e}_{R_{k}}$. It still remains true that $\operatorname{ker} \theta_{k}=\bigcap_{P \in \mathfrak{P}_{k}} P$.

It follows from the result of Bade and Curtis that $\mathcal{I}\left(\theta_{k}\right)=\operatorname{ker} \theta_{k}$ when $0 \leq k \leq n$. Thus the homomorphism $\theta: \mathcal{C}_{0}(\Omega) \rightarrow \prod_{k=0}^{n} R_{k}^{\#}$ defined by $\theta(a)=$ $\left(\theta_{0}(a), \ldots, \theta_{n}(a)\right)$ satisfies

$$
\mathcal{I}(\theta)=\bigcap_{k=0}^{n} \mathcal{I}\left(\theta_{k}\right)=\bigcap_{P \in \mathfrak{P}} P=I,
$$

as required. 
REMARK 6.9. Let $I$ be the intersection of a relatively compact family $\mathfrak{P}$ of prime ideals in $\mathcal{C}_{0}(\Omega)$. Suppose that $I$ is also the intersection of a countable family of prime ideals. Denote by $\mathfrak{P}_{*}$ the set of minimal elements in $\mathfrak{P}$. Then $\mathfrak{P}_{*}$ is also relatively compact, and $I=\bigcap_{P \in \mathfrak{P}_{*}} P$, by Proposition 5.5(iii). Since $I$ is the intersection of a countable family of prime ideals, we see that $\mathfrak{P}_{*}$ is countable (using Lemma 5.6). Since $\mathfrak{P}_{*}$ is countable, it is obvious that every chain in the closure of $\mathfrak{P}_{*}$ is countable.

\section{Examples on metrizable locally compact spaces}

For examples of pseudofinite sequences of prime ideals in $\mathcal{C}_{0}(\Omega)$, see [15]. In this section, we shall construct relatively compact families of prime ideals that are not unions of finitely many pseudofinite families.

For the entire section, fix a well-ordered set $\kappa$. Set $\kappa^{(0)}=\kappa$. For all $n \in \mathbb{N}$, define $\kappa^{(n)}$ inductively as the set of limiting elements in $\kappa^{(n-1)}$. We shall only consider those $\kappa$ for which $\kappa^{(n)}=\emptyset$ for some $n \in \mathbb{N}$. This condition forces $\kappa$ to be countable. Let $d$ be the largest integer for which $\kappa^{(d)} \neq \emptyset$; we call it the depth of $\kappa$. For simplicity, we also suppose that $\kappa$ has the largest element, $\max \kappa$, and that $\kappa^{(d)}=\{\max \kappa\}$. Otherwise, we can always replace $\kappa$ by a bigger well-ordered set.

For all $\alpha \in \kappa$ define $l(\alpha)$ to be the largest integer $l$ for which $\alpha \in \kappa^{(l)}$. We define a relation $\prec$ on $\kappa$ as follows: for all $\alpha, \beta \in \kappa$, we write $\alpha \prec \beta$ if $\beta$ is the smallest element in $\kappa$ such that $\beta \geq \alpha$ and $l(\beta)=l(\alpha)+1$. We then define a partial order $\ll$ on $\kappa$ as follows: for all $\alpha, \beta \in \kappa$, we write $\alpha \ll \beta$ if there exists a finite sequence $\alpha=\gamma_{1} \prec \gamma_{2} \prec \cdots \prec \gamma_{n}=\beta$. Note that, for each $\alpha \in \kappa$, there is exactly one chain $\alpha=\gamma_{1} \prec \gamma_{2} \prec \cdots$, which must be finite, and the ultimate end point of this chain is $\max \kappa$. We also set

$$
\kappa_{0}=\{\beta \in \kappa \mid l(\beta)=0\}
$$

LEMMA 7.1. The relation $\ll$ on the well-ordered set $\kappa$ has the following properties.

(i) If $\beta \ll \alpha$ and $\alpha \in \kappa_{0}$ then $\beta=\alpha$.

(ii) If $\gamma \ll \alpha$ and $\gamma \ll \beta$ then either $\alpha \ll \beta$ or $\beta \ll \alpha$.

(iii) If $\beta \ll \alpha$ and $\beta \leq \gamma \leq \alpha$ then $\gamma \ll \alpha$.

(v) If $\beta \ll \alpha$ and $\beta \neq \alpha$ then there exists $\gamma \in \kappa_{0}$ such that $\beta<\gamma<\alpha$ (and so $\gamma \ll \alpha)$.

PROOF. The proof is routine.

We start our construction with the following general lemma.

LEMMA 7.2. Let A be a commutative algebra and $Q$ be an ideal that either is prime in $A$ or is $A$ itself. Suppose that we have $\left(f_{\alpha}: \alpha \in \kappa\right) \subseteq Q$ and a semiprime ideal $I \subseteq Q$ with the following properties.

(i) $f_{\alpha} \notin I$ and $I: f_{\alpha} \subseteq Q$ for all $\alpha \in \kappa$.

(ii) $f_{\alpha} f_{\beta} \in I$ whenever both $\alpha \ll \beta$ and $\beta \ll \alpha$ in $\kappa$. 
(iii) If $g f_{\alpha} \in I$ for some $\alpha \in \kappa$ then $g f_{\beta} \in I$ for all $\beta \ll \alpha$.

(iv) If $g f_{\beta_{0}} \in I$ for some $\beta_{0} \in \kappa_{0}$, then, whenever $\alpha \gg \beta_{0}$, there exists $\beta_{1} \in \kappa_{0}$ with $\beta_{1} \ll \alpha$ such that $g f_{\beta} \in I$ for all $\beta \in \kappa_{0}$ with $\beta_{1} \leq \beta \leq \alpha$.

Then there exist prime ideals $P_{\alpha}$, where $\alpha \in \kappa$, such that:

(a) $f_{\alpha} \notin P_{\alpha}$ and $I: f_{\alpha} \subseteq P_{\alpha} \subseteq Q$ for all $\alpha \in \kappa$;

(b) $P_{\alpha}=\bigcup_{\beta \ll \alpha, \beta \neq \alpha} P_{\beta}$ for all $\alpha \in \kappa$;

(c) if $g \in P_{\alpha}$ for some $\alpha \in \kappa$ then there exists $\beta_{1} \in \kappa_{0}$ with $\beta_{1} \ll \alpha$ such that $g \in P_{\beta}$ for all $\beta \in \kappa_{0}$ with $\beta_{1} \leq \beta \leq \alpha$.

PROOF. We prove the lemma by induction on the depth $d$ of $\kappa$.

If $d=0$, then $\kappa=\{0\}$. Conditions (i) to (iv) reduce to $I$ being semiprime, $f_{0} \notin I$ and $I: f_{0} \subseteq Q$. It follows that $I \cap S=\emptyset$, where $S=\left\{f_{0}^{k}, f_{0}^{k} f \mid k \geq 1, f \in A \backslash Q\right\}$ is closed under multiplication. Therefore, we can find a prime ideal $P_{0}$ such that $I_{0} \subseteq P_{0}$ and $P_{0} \cap S=\emptyset$, and so $P_{0} \subseteq Q$ and $f_{0} \notin P_{0}$. We see that $P_{0}$ is the required prime ideal.

Now, suppose that the result holds whenever the depth is less than $d$. By Zorn's lemma, we can find a semiprime ideal $J$ containing $I$ such that $J$ is maximal with respect to conditions (i) to (iv).

Claim 1: If $f \notin Q$ then $J: f=J$. Indeed, it is clear that $J: f$ is semiprime and satisfies conditions (i) to (iv). So the maximality of $J$ implies $J: f=J$.

Claim 2: If $f \notin J$ then $J: f \subseteq Q$. For otherwise, there would exist $g \in J: f \backslash Q$, and so $f \in J: g=J$, by Claim 1 ; this is a contradiction.

Set $P=\bigcup_{\alpha \in \kappa} J: f_{\alpha}$. Then $P \subseteq Q$. Condition (iii) implies that

$$
P=\bigcup_{\alpha \in \kappa_{0}} J: f_{\alpha}
$$

and condition (iv) implies that $P$ is an ideal, by choosing $\alpha=\max \kappa$.

Claim 3: If $f \notin P$ then $J: f=J$. Indeed, it is easy to see that $J: f$ is semiprime and satisfies conditions (i) to (iv) (the least obvious one is (i), however, since $f \notin P$, $f f_{\alpha} \notin J$, and so $f_{\alpha} \notin J: f$ and $(J: f): f_{\alpha}=J: f f_{\alpha} \subseteq Q$ by Claim 2). So the maximality of $J$ implies $J: f=J$.

Claim 4: $P$ is either prime in A or A itself. Indeed, if $f, g \notin P$, then, by Claim 3,

$$
g \notin \bigcup_{\alpha \in \kappa} J: f_{\alpha}=\bigcup_{\alpha \in \kappa}(J: f): f_{\alpha}=P: f .
$$

Thus $f g \notin P$.

Let $\alpha_{1}<\alpha_{2}<\cdots$ be the nonlimiting elements in $\kappa^{(d-1)}$; their limit is max $\kappa$. Set $\kappa_{1}=\left\{\alpha \in \kappa \mid \alpha \leq \alpha_{1}\right\}$, and $\kappa_{n}=\left\{\alpha \in \kappa \mid \alpha_{n-1}<\alpha \leq \alpha_{n}\right\}$ for all $n \geq 2$. Each $\kappa_{n}$ has depth $d-1$, and $\kappa=\bigcup_{n=1}^{\infty} \kappa_{n} \cup\{\max \kappa\}$.

For all $n \in \mathbb{N}$, we see that $\left(f_{\alpha}: \alpha \in \kappa_{n}\right), J$, and $P$ are such that $\left(f_{\alpha}: \alpha \in \kappa_{n}\right) \subseteq P$ and $J \subseteq P$, and conditions (i) to (iv) hold, with $\kappa_{n}$ replacing $\kappa, J$ replacing $I$, and $P$ 
replacing $Q$. So, by induction, there exist prime ideals $P_{\alpha}$, where $\alpha \in \kappa_{n}$, satisfying conditions (a) to (c) (with obvious modifications). Set $P_{\max \kappa}=P$.

Note that if $\beta \ll \alpha<\max \kappa$ then both $\alpha$ and $\beta$ belong to the same $\kappa_{n}$ for some $n \in \mathbb{N}$. We see that the combined family $\left(P_{\alpha}: \alpha \in \kappa\right)$ obviously satisfies conditions (a) to (c) (with $J$ replacing $I$ ); the only one that we really need to check is condition (c) when $\alpha=\max \kappa$, but this follows from the facts that $J: \beta \subseteq P_{\beta} \subseteq P_{\max \kappa}$, that

$$
P_{\max \kappa}=\bigcup_{\beta \in \kappa_{0}} J: f_{\beta},
$$

and that $J$ satisfies condition (iv).

Now, let $\Omega$ be a metrizable locally compact space. We define a nonincreasing sequence $\left(\partial^{(n)} \Omega^{b}: n \in \mathbb{Z}^{+}\right)$of compact subsets of $\Omega^{b}$ as follows:

(i) put $\partial^{(0)} \Omega^{b}=\Omega^{b}$;

(ii) for all $n \in \mathbb{Z}^{+}$, define $\partial^{(n+1)} \Omega^{\text {b }}$ to be the set of all limit points of $\partial^{(n)} \Omega^{b}$.

Define $\partial^{(\infty)} \Omega^{b}=\bigcap_{n \in \mathbb{Z}^{+}} \partial^{(n)} \Omega^{b}$. By the compactness, either $\partial^{(\infty)} \Omega^{b}$ is nonempty or $\partial^{(l)} \Omega^{b}$ is empty for some $l \in \mathbb{Z}^{+}$.

To construct nontrivial pseudofinite sequences of prime ideals in $\mathcal{C}_{0}(\Omega)$ it is necessary that there exists $p \in \partial^{(\infty)} \Omega^{b}$; this follows from [15, Proposition 8.7]. Remark that all uncountable Polish spaces possess such a point $p$, and there are even countable compact subspaces of $\mathbb{R}$ satisfying this condition.

We need some further preparations. For each $\beta \in \kappa \backslash\{\max \kappa\}$, there exists a unique $\alpha \in \kappa$ such that $\beta \prec \alpha$. We define $t(\beta)$ to be the number of $\gamma \in \kappa$ such that $\gamma \prec \alpha$ and $\gamma \leq \beta$; there are only finitely many such $\gamma$. For each $\beta \in \kappa_{0}$, there exists a unique $\left(\alpha_{1}, \ldots, \alpha_{d-1}\right) \in \kappa$ such that

$$
\beta \prec \alpha_{1} \prec \cdots \prec \alpha_{d-1} \prec \max \kappa ;
$$

set $w(\beta)=\max \left\{t(\beta), t\left(\alpha_{1}\right), \ldots, t\left(\alpha_{d-1}\right)\right\}$. For all $k \in \mathbb{N}$,

$$
\left|\left\{\alpha \in \kappa_{0} \mid w(\alpha) \leq k\right\}\right|=k^{d} .
$$

Adjoin $\infty$ to $\mathbb{N}$ to obtain its one-point compactification $\mathbb{N}^{b}$; the convention is that $\infty>n$ for all $n \in \mathbb{N}$ and $2^{-\infty}=0$. Define $\Xi$ to be the subset of the product space $\left(\mathbb{N}^{b}\right)^{\kappa_{0}}$ consisting of all elements $\left(n_{\alpha}: \alpha \in \kappa_{0}\right)$ with the property that there exists a finite set $F \subseteq \kappa_{0}$ such that $n_{\alpha}=\infty$ when $\alpha \in \kappa_{0} \backslash F$ and such that $n_{\alpha} \geq \max \{w(\beta) \mid \beta \in F\}$ when $\alpha \in F$. It is easy to see that $\Xi$ is a closed subset of $\left(\mathbb{N}^{b}\right)^{\kappa_{0}}$.

LEMMA 7.3. Let $\Omega$ be a metrizable locally compact space and $p \in \partial^{(\infty)} \Omega^{b}$. Then the space $\Xi$ can be continuously embedded into $\Omega^{b}$ such that the point $\infty=(\infty, \infty, \ldots)$ is mapped into $p$.

PROOF. As shown in [15, Lemmas 9.1 and 9.2], the compact subset

$$
\Delta=\{0\} \cup\left\{\sum_{i=1}^{k} 2^{-n_{i}} \mid k, n_{1}, n_{2}, \ldots, n_{k} \in \mathbb{N} \text { and } k \leq n_{1}<\cdots<n_{k}\right\}
$$

of $\mathbb{R}$ is continuously embedded in $\Omega^{b}$ such that 0 is mapped into $p$. 
Choose an injective map $r: \kappa_{0} \times \mathbb{N} \rightarrow \mathbb{N}$ such that $r(\alpha, j) \geq j^{d}$ for all $\alpha \in \kappa_{0}$ and $j \in \mathbb{N}$; this is possible since $\kappa_{0}$ is countable. For convenience, for all $\alpha \in \kappa_{0}$, we also set $r(\alpha, \infty)=\infty$. Define a map $\tau$ from $\Xi$ into $[0,1]$ as follows: for all $\left(n_{\alpha}\right)_{\alpha \in \kappa_{0}} \in \Xi$, set

$$
\tau:\left(n_{\alpha}\right)_{\alpha \in \kappa_{0}} \mapsto \sum_{\alpha \in \kappa_{0}} 2^{-r\left(\alpha, n_{\alpha}\right)}
$$

Then we see that $\tau$ is well defined, injective and continuous.

Let $\left(n_{\alpha}\right)_{\alpha \in \kappa_{0}} \in \Xi$ be arbitrary. Set $F=\left\{\alpha \in \kappa_{0} \mid n_{\alpha}<\infty\right\}$. Then $F$ is a finite subset of $\kappa_{0}$, and $n_{\alpha} \geq k=\max \{w(\beta) \mid \beta \in F\}$ for all $\alpha \in F$. So we see that, for $\alpha \in F$,

$$
r\left(\alpha, n_{\alpha}\right) \geq n_{\alpha}^{d} \geq k^{d}=\left|\left\{\beta \in \kappa_{0} \mid w(\beta) \leq k\right\}\right| \geq|F| .
$$

From this, we deduce that $\tau$ maps $\Xi$ into $\Delta$, and the lemma follows.

LEMMA 7.4. Let $\Omega$ be a metrizable locally compact space, and let $p \in \partial^{(\infty)} \Omega^{b}$. Then there exist a family of prime ideals $\left(P_{\alpha}: \alpha \in \kappa\right)$ in $\mathcal{C}_{0}(\Omega)$, where each ideal is supported at $p$, and a family of functions $\left(f_{\alpha}: \alpha \in \kappa\right)$ in $\mathcal{C}_{0}(\Omega)$ such that:

(a) $f_{\alpha} \notin P_{\alpha}$ and $f_{\beta} \in P_{\alpha}$ whenever both $\beta \ll \alpha$ and $\alpha \ll \beta$;

(b) $P_{\alpha}=\bigcup_{\beta \ll \alpha, \beta \neq \alpha} P_{\beta}$ for all $\alpha \in \kappa$;

(c) for all $\alpha \in \kappa$, if $g \in P_{\alpha}$ then there exists $\beta_{1} \in \kappa_{0}$ with $\beta_{1} \ll \alpha$ such that $g \in P_{\beta}$ for all $\beta \in \kappa_{0}$ with $\beta_{1} \leq \beta \leq \alpha$.

PROOF. It follows from the previous lemma that we only need to consider the case where $\Omega=\Xi$ and $p=\infty$. Thus suppose that $\Omega=\Xi$ and $p=\infty$.

For each $\alpha \in \kappa_{0}$ define

$$
Z_{\alpha}=\left\{\left(n_{\beta}\right)_{\beta \in \kappa_{0}} \in \Xi \mid n_{\alpha}=\infty\right\}
$$

and for all $\alpha \in \kappa \backslash \kappa_{0}$ define

$$
Z_{\alpha}=\bigcap_{\substack{\beta \in \kappa_{0} \\ \beta \ll \alpha}} Z_{\beta} .
$$

Then choose $f_{\alpha} \in \mathcal{C}(\Xi)$ such that $Z_{\alpha}=\mathbf{Z}\left(f_{\alpha}\right)$. Let $\mathcal{F}$ be the $z$-filter generated by all $Z_{\alpha} \cup Z_{\beta}$ where $\alpha, \beta \in \kappa, \alpha \nless \beta$ and $\beta \ll \alpha$. Then define $I=\mathbf{Z}^{-1}[\mathcal{F}]$. Obviously $I$ is a semiprime ideal, $I \subseteq M_{\infty}$, and $\left(f_{\alpha}: \alpha \in \kappa\right) \subseteq M_{\infty}$.

It is sufficient to prove that $\left(f_{\alpha}\right), I$, and $\bar{M}_{\infty}$ satisfy conditions (i) to (iv) of Lemma 7.2.

First, for all $\gamma \in \kappa$, we see that $f \in I: f_{\gamma}$ if and only if

$$
\mathbf{Z}(f) \cup Z_{\gamma} \supseteq \bigcap_{k=1}^{n}\left(Z_{\alpha_{k}} \cup Z_{\beta_{k}}\right),
$$

where $\alpha_{k} \ll \beta_{k}$ and $\beta_{k} \ll \alpha_{k}$ for each $k$. We see from Lemma 7.1 that, for all $k$, one of the following three cases must happen:

(1) $\alpha_{k} \ll \gamma$ and $\gamma \ll \alpha_{k}$;

(2) $\gamma \ll \beta_{k}$ and $\beta_{k} \ll \gamma$;

(3) $\alpha_{k} \ll \gamma, \beta_{k} \ll \gamma, \alpha_{k} \ll \beta_{k}$ and $\beta_{k} \ll \alpha_{k}$. 
Thus we see that $f \in I: f_{\gamma}$ implies that

$$
\mathbf{Z}(f) \cup Z_{\gamma} \supseteq \bigcap_{i=1}^{r} Z_{\varrho_{i}} \cap \bigcap_{j=1}^{s}\left(Z_{\sigma_{j}} \cup Z_{\zeta_{j}}\right),
$$

where $\varrho_{i} \ll \gamma$ and $\gamma \ll \varrho_{i}$ for all $i$, and $\sigma_{j} \ll \gamma, \zeta_{j} \ll \gamma, \sigma_{j} \ll \zeta_{j}$ and $\zeta_{j} \ll \sigma_{j}$ for all $j$. In particular, we see that $\sigma_{j}<\gamma$ whenever $1 \leq j \leq s$, and so, by Lemma 7.1(iv), there exists $\beta \in \kappa_{0}$ such that

$$
\beta>\max \left\{\sigma_{j} \mid 1 \leq j \leq s\right\} \quad \text { and } \quad \beta \ll \gamma .
$$

These also imply that $\beta \ll \sigma_{j}$ if $1 \leq j \leq s$ and $\beta \ll \varrho_{i}$ if $1 \leq i \leq r$. Thus for all $k \geq w(\beta)$, we set $n_{\beta}^{(k)}=k$ and $n_{\alpha}^{(k)}=\infty$ when $\alpha \neq \beta$, and then

$$
\left(n_{\alpha}^{(k)}: \alpha \in \kappa_{0}\right) \in\left(\bigcap_{i=1}^{r} Z_{\varrho_{i}} \cap \bigcap_{j=1}^{s} Z_{\sigma_{j}}\right) \backslash Z_{\gamma} \subseteq \mathbf{Z}(f) .
$$

On the other hand, $\lim _{k \rightarrow \infty}\left(n_{\alpha}^{(k)}: \alpha \in \kappa_{0}\right)=\infty$ in $\Xi$. It follows that $\infty \in \mathbf{Z}(f)$. Hence, $I: f_{\gamma} \subseteq M_{\infty}$ and $f_{\gamma} \notin I$, so condition (i) of Lemma 7.2 holds.

It is obvious that conditions (ii) and (iii) of Lemma 7.2 are satisfied by the definitions of $I$ and the sets $Z_{\alpha}$.

Now, let $\beta_{0}, \alpha \in \kappa$ and let $g \in \mathcal{C}(\Xi)$ be such that $l\left(\beta_{0}\right)=0, \beta_{0} \ll \alpha, \beta_{0} \neq \alpha$ (these imply that $\left.\alpha \notin \kappa_{0}\right)$ and $g f_{\beta_{0}} \in I$. Then, from the previous discussion, noting that $l\left(\beta_{0}\right)=0$,

$$
\mathbf{Z}(g) \cup Z_{\beta_{0}} \supseteq \bigcap_{i=1}^{r} Z_{\varrho_{i}} \quad \text { or } \quad \mathbf{Z}(g) \supseteq \overline{\bigcap_{i=1}^{r} Z_{\varrho_{i}} \backslash Z_{\beta_{0}}},
$$

where $\beta_{0} \nless \varrho_{i}$, which implies that $\alpha \ll \varrho_{i}$ for all $i$. We claim that

$$
\mathbf{Z}(g) \supseteq \bigcap_{i=1}^{r} Z_{\varrho_{i}} \cap \bigcap_{\substack{\gamma \in \kappa_{0} \\ w(\gamma) \leq w\left(\beta_{0}\right)}} Z_{\gamma} .
$$

Indeed, let $\left(n_{\gamma}: \gamma \in \kappa_{0}\right) \neq \infty$ be in the right-hand-side set. Then, $n_{\gamma}=\infty$ whenever $\gamma \ll \varrho_{i}$ for some $i$ or $w(\gamma) \leq w\left(\beta_{0}\right)$ for all $\gamma \in \kappa_{0}$. Set $F=\left\{\gamma \in \kappa_{0} \mid n_{\gamma}<\infty\right\}$. Then $F$ is a nonempty finite set and

$$
n_{\gamma} \geq \max \{w(\beta) \mid \beta \in F\}>w\left(\beta_{0}\right) \quad \forall \gamma \in F .
$$

Thus by setting $n_{\beta_{0}}^{(k)}=k$ when $k \geq \max \{w(\beta) \mid \beta \in F\}$ and $n_{\gamma}^{(k)}=n_{\gamma}$ when $\gamma \neq \beta_{0}$, we obtain

$$
\left(n_{\gamma}^{(k)}: \gamma \in \kappa_{0}\right) \in \bigcap_{i=1}^{r} Z_{\varrho_{i}} \backslash Z_{\beta_{0}} \subseteq \mathbf{Z}(g) .
$$

On the other hand, we see that $\lim _{k \rightarrow \infty}\left(n_{\gamma}^{(k)}: \gamma \in \kappa_{0}\right)=\left(n_{\gamma}: \gamma \in \kappa_{0}\right)$ in $\Xi$. It follows that $\left(n_{\gamma}: \gamma \in \kappa_{0}\right) \in \mathbf{Z}(g)$. The claim follows. 
Set

$$
A=\left\{\gamma \in \kappa_{0} \mid \gamma \ll \alpha \text { and } w(\gamma) \leq w\left(\beta_{0}\right)\right\}
$$

and

$$
B=\left\{\varrho_{i} \mid 1 \leq i \leq r, \varrho_{i} \ll \alpha\right\} .
$$

By Lemma 7.1(iv), we can choose $\beta_{1} \in \kappa_{0}$ such that $\beta_{1} \ll \alpha$ and

$$
\beta_{1}>\max (A \cup B) ;
$$

note that $\max (A \cup B) \ll \alpha$ and $\max (A \cup B) \neq \alpha$. Let $\beta \in \kappa_{0}$ be such that $\beta_{1} \leq \beta \leq \alpha$. Then $\beta \ll \alpha$. We see that $\varrho_{i} \ll \beta$ when $1 \leq i \leq r$; otherwise we see that $\varrho_{i}=\beta \ll \alpha$ but then $\beta \geq \beta_{1}>\varrho_{i}$ by (7.1), contradicting $\varrho_{i}=\beta$. We also see that $\beta \ll \varrho_{i}$ when $1 \leq i \leq r$; otherwise either $\varrho_{i} \ll \alpha$ or $\alpha \ll \varrho_{i}$, and we have already ruled out $\alpha \ll \varrho_{i}$ from the beginning of the previous paragraph, so $\varrho_{i} \ll \alpha$, and again, this and (7.1) imply that $\beta \geq \beta_{1}>\varrho_{i}$, contradicting $\beta \ll \varrho_{i}$. We also see that $\beta \ll \gamma$ and $\gamma \ll \beta$ for all $\gamma \in \kappa_{0}$ with $w(\gamma) \leq w\left(\beta_{0}\right)$; otherwise $\gamma=\beta \ll \alpha$. However, this and (7.1) imply that $\beta \geq \beta_{1}>\gamma$. Thus

$$
\mathbf{Z}(g) \cup Z_{\beta} \supseteq \bigcap_{i=1}^{r}\left(Z_{\varrho_{i}} \cup Z_{\beta}\right) \cap \bigcap_{\substack{\gamma \in \kappa_{0} \\ w(\gamma) \leq w\left(\beta_{0}\right)}} Z_{\gamma} \cup Z_{\beta} \in \mathcal{F} ;
$$

and so $g f_{\beta} \in I$. Hence, condition (iv) of Lemma 7.2 is also satisfied.

We now state the main theorem of this section.

THEOREM 7.5. Suppose that $p \in \partial^{(\infty)} \Omega^{b}$ where $\Omega$ is a metrizable locally compact space, and that $\kappa$ is a well-ordered set as above. Then there exists a compact family of prime ideals $\left(P_{\alpha}: \alpha \in \kappa\right)$ in $\mathcal{C}_{0}(\Omega)$, where each ideal is supported at $p$, satisfying the following conditions.

(a) $\quad\left(P_{\alpha_{n}}: n \geq n_{0}\right)$ is a pseudofinite sequence with union $P_{\alpha}$ for some $n_{0} \in \mathbb{N}$ whenever $\left(\alpha_{n}\right)$ converges to $\alpha$ in the order topology of $\kappa$.

(b) $P_{\alpha} \subseteq P_{\beta}$ if and only if $\alpha \ll \beta$.

Let $\mathfrak{P}$ be any relatively compact family of prime ideals with the same intersection as $\bigcap_{\alpha \in \kappa} P_{\alpha}$. Then the closure of $\mathfrak{P}$ contains a chain of length $d+1$. In particular, $\mathfrak{P}$ is not the union of any finite collection of many pseudofinite subfamilies of prime ideals when $d>1$.

PROOF. Let $\left(P_{\alpha}: \alpha \in \kappa\right)$ be the family constructed in Lemma 7.4.

(a) Without loss of generality, we assume that $\alpha_{n} \neq \alpha$ for all $n \in \mathbb{N}$. There exists $n_{0} \in \mathbb{N}$ such that $\alpha_{n} \ll \alpha$ for all $n \geq n_{0}$. We see that $P_{\alpha_{n}} \subseteq P_{\alpha}$ for all $n \geq n_{0}$ and

$$
P_{\alpha}=\bigcup_{\substack{\beta \in \kappa_{0} \\ \beta \ll \alpha}} P_{\beta}
$$

So by Theorem 7.5(c), for each $g \in P_{\alpha}$, there exists $\beta_{1} \in \kappa_{0}$ with $\beta_{1} \ll \alpha$ such that $g \in P_{\beta}$ for all $\beta \in \kappa_{0}$ with $\beta_{1} \leq \beta \leq \alpha$. Choose $n_{1} \geq n_{0}$ such that $\alpha_{n} \geq \beta_{1}$ for all $n \geq n_{1}$. Let $n \geq n_{1}$ be arbitrary. Pick $\beta^{\prime} \in \kappa_{0}$ such that $\beta^{\prime} \ll \alpha_{n}$. If $\beta^{\prime} \leq \beta_{1}$, 
then $\beta_{1} \ll \alpha_{n}$ by Lemma 7.1(iii), and so $g \in P_{\beta_{1}} \subseteq P_{\alpha_{n}}$. Otherwise $\beta_{1}<\beta^{\prime}$, then $g \in P_{\beta^{\prime}} \subseteq P_{\alpha_{n}}$. Thus $\left(P_{\alpha_{n}}: n \geq n_{0}\right)$ is a pseudofinite sequence whose union is $P_{\alpha}$.

(b) This is a consequence of Lemma 7.4(a) and (b).

In the order topology, $\kappa$ is a compact metrizable space; each sequence in $\kappa$ has a convergence subsequence. The compactness of $\left\{P_{\alpha} \mid \alpha \in \kappa\right\}$ thus follows from (a).

Let $\mathfrak{P}$ be a relatively compact family of prime ideals such that

$$
\bigcap_{P \in \mathfrak{P}} P=I=\bigcap_{\alpha \in \kappa} P_{\alpha} .
$$

Denote by $\mathfrak{P}_{*}$ the subfamily consisting of minimal elements of $\mathfrak{P}$. By Proposition 5.5(iii), we see that $\bigcap_{P \in \mathfrak{P}_{*}} P=I$. Lemma 5.6 shows that, for each $P \in \mathfrak{P}_{*}$ there exists $f_{P} \notin P$ but $f_{P} \in Q$ for all $Q \in \mathfrak{P}_{*} \backslash\{P\}$; in particular, $P=I: f_{P}$. This and Lemma 4.8 then imply that $\mathfrak{P}_{*}$ is exactly the set of prime ideals of the form $I: f$ for some $f \in \mathcal{C}_{0}(\Omega)$. Similarly, $\left\{P_{\alpha} \mid \alpha \in \kappa_{0}\right\}$ is also exactly the set of prime ideals of the form $I: f$ for some $f \in \mathcal{C}_{0}(\Omega)$. Thus

$$
\mathfrak{P}_{*}=\left\{P_{\alpha} \mid \alpha \in \kappa_{0}\right\} \quad \text { and its closure is }\left\{P_{\alpha} \mid \alpha \in \kappa\right\} .
$$

Therefore, whenever $\alpha_{0} \prec \alpha_{1} \prec \cdots \prec \alpha_{d}=\max \kappa$, we have a chain of length $d+1$ in the closure of $\mathfrak{P}_{*}$, namely $P_{\alpha_{0}} \subset \cdots \subset P_{\alpha_{d}}$. Obviously, this shows that $\mathfrak{P}$ cannot be the union of any finite number of pseudofinite families when the depth of $\kappa$ is larger than 1 .

REMARK 7.6. Since the cardinality of $\mathcal{C}(\Xi)$ is $\mathfrak{c}$, we see that, for $\left(P_{\alpha}: \alpha \in \kappa\right)$ as in the above theorem,

$$
\left|\mathcal{C}_{0}(\Omega) / \bigcap_{\alpha \in \kappa} P_{\alpha}\right|=\mathfrak{c} .
$$

Let $\Omega$ be any metrizable locally compact space with $\partial^{(\infty)} \Omega \neq \emptyset$. Let $\mathfrak{P}$ be a relatively compact family of prime ideals in $\mathcal{C}_{0}(\Omega)$. Since we are mostly interested in $\bigcap_{P \in \mathfrak{P}} P$, by passing to the minimal elements of $\mathfrak{P}$, we can suppose that $P \not \subset Q$ for each distinct $P, Q \in \mathfrak{P}$. Let us call a family satisfying this condition reduced.

QUESTION. Is there a reduced relatively compact family of prime ideals $\mathfrak{P}$ in $\mathcal{C}_{0}(\Omega)$ whose closure contains an uncountable chain or (merely) an infinite chain? If there exists such a reduced family $\mathfrak{P}$ whose closure contains an uncountable chain, is it still possible to remove the countability condition from Theorems 6.7 and 6.8?

We know that every chain in the closure of a relatively compact family of prime ideals must be well ordered (Proposition 5.5(iii)). However, we show in [16] the existence of uncountable well-ordered chains of prime ideals in $\mathcal{C}_{0}(\Omega)$. We also show in [16] that there is an uncountable, nonredundant, pseudofinite family of prime ideals in $\mathcal{C}_{0}(\Omega)$, thus, in particular, showing that there exists an uncountable, reduced, relatively compact family of prime ideals (however, every chain in the closure of a pseudofinite family of prime ideals has length at most 2). 


\section{Acknowledgement}

The author would like to thank Professor Anthony To-Ming Lau for his generous support and encouragement during this research.

\section{References}

[1] W. G. Bade and P. C. Curtis Jr, 'Homomorphisms of commutative Banach algebras', Amer. J. Math. 82 (1960), 589-608.

[2] H. G. Dales, 'A discontinuous homomorphism from $C(X)$ ', Amer. J. Math. 101 (1979), 647-734.

[3] H. G. Dales, Banach Algebras and Automatic Continuity, London Mathematical Society Monographs Series, 24 (Clarendon Press, Oxford, 2000).

[4] H. G. Dales and W. H. Woodin, An Introduction to Independence for Analysts, London Mathematical Society Lecture Note Series, 115 (Cambridge University Press, Cambridge, 1987).

[5] H. G. Dales and W. H. Woodin, Super-Real Fields: Totally Ordered Fields with Additional Structure, London Mathematical Society Monographs Series, 14 (Clarendon Press, Oxford, 1996).

[6] J. R. Esterle, 'Seminormes sur C(K)', Proc. London Math. Soc. (3) 36 (1978), 27-45.

[7] J. R. Esterle, 'Sur l'existence d'un homomorphisme discontinu de $C(K)$ ', Proc. London Math. Soc. (3) 36 (1978), 46-58.

[8] J. R. Esterle, 'Injection de semi-groupes divisibles dans des algèbres de convolution et construction d'homomorphismes discontinus de $C(K)^{\prime}$ ', Proc. London Math. Soc. (3) 36 (1978), 59-85.

[9] J. R. Esterle, 'Homomorphismes discontinus des algèbres de Banach commutatives séparables', Studia Math. 66 (1979), 119-141.

[10] J. R. Esterle, 'Universal properties of some commutative radical Banach algebras', J. Reine Angew. Math. 321 (1981), 1-24.

[11] L. Gillman and M. Jerison, Rings of Continuous Functions, University Series in Higher Mathematics (D. Van Nostrand, Princeton, NJ, 1960).

[12] B. E. Johnson, 'Norming $C(U)$ and related algebras', Trans. Amer. Math. Soc. 220 (1976), 37-58.

[13] I. Kaplansky, 'Normed algebras', Duke Math. J. 16 (1949), 399-418.

[14] K. Kendig, Elementary Algebraic Geometry, Graduate Texts in Mathematics, 44 (Springer, New York, 1977).

[15] H. L. Pham, 'The kernels of radical homomorphisms and intersections of prime ideals', Trans. Amer. Math. Soc. 360 (2008), 1057-1088.

[16] H. L. Pham, 'Uncountable families of prime $z$-ideals in $\mathcal{C}_{0}(\mathbb{R})^{\prime}$, Bull. London Math. Soc. 41 (2009), 354-366.

[17] A. M. Sinclair, 'Homomorphisms from $C_{0}(R)$ ', J. London Math. Soc. (2) 11 (1975), 165-174.

[18] A. M. Sinclair, Automatic Continuity of Linear Operators, London Mathematical Society Lecture Note Series, 21 (Cambridge University Press, Cambridge, 1976).

HUNG LE PHAM, Department of Mathematical and Statistical Sciences, University of Alberta, Edmonton, Alberta, Canada T6G 2G1

and

Current address: School of Mathematics, Statistics, and Operations Research, Victoria University, PO Box 600, Wellington 6140, New Zealand e-mail: Hung.Pham@vuw.ac.nz 